\title{
Das schwer verletzte Kind
}

\author{
B. Auner, H. Jakob, I. Marzi \\ Klinik für Unfall-, Hand- und Wiederherstellungschirurgie, Universitätsklinikum Frankfurt am Main
}

Zwar ist das Polytrauma des Kindes insgesamt selten. Auch große Traumazentren weisen eine nur geringe Fallzahl schwerstverletzter Kinder auf. Und dennoch bleibt der Unfall seit Jahrzehnten die häufigste Todesursache im frühen Kindes- und Jugendalter in Europa.

In Deutschland wird die Bildung sogenannter kindertraumatologischer Kompetenzzentren diskutiert, da bereits die primäre Behandlung in einem Zentrum, das über die geeignete Kinderexpertise verfügt, stattfinden sollte. Doch abhängig von den örtlichen Gegebenheiten und der Dringlichkeit kommen auch kleinere Zentren zumindest für die Primärstabilisierung infrage. Die Verlegungskriterien wurden erstmals im Weißbuch Schwerverletztenversorgung der Deutschen Gesellschaft für Unfallchirurgie (DGU) festgelegt.

\section{Einleitung}

Für eine adäquate Behandlung müssen anatomische und physiologische Besonderheiten beim Kind berücksichtigt werden, ebenso sind Kenntnisse über mögliche Verletzungsursachen und Verletzungsmuster sowie deren Häufigkeit von großer Bedeutung. Je jünger die Kinder sind, desto deutlicher treten Unterschiede im Verletzungsmuster sowie in der Schwere der Verletzung der betroffenen Organsysteme zum Erwachsenen auf.

Eine inadäquate Primärbeurteilung kann für frühe Todesfälle verantwortlich sein.

Prognoseentscheidend ist in erster Linie die Schwere des Schädel-Hirn-Traumas.

\section{Epidemiologie und Ätiologie}

Ein relativ geringer Anteil von 5\% aller polytraumatisierten Patienten sind Kinder unter 15 Jahren. Trotz stetig sinkender Zahlen bleiben Unfälle die häufigste Todesursache bei Kindern und Jugendlichen. In Deutschland starben im Jahr 2012 laut Statistischem Bundesamt 308 Kinder unter 15 Jahren, was 3 Todesfällen je 100000 Kinder entspricht.

\section{Abkürzungen}

$\begin{array}{ll}\text { AIS } & \text { Abbreviated Injury Scale } \\ \text { AO } & \text { Arbeitsgemeinschaft für Osteosynthesefragen } \\ \text { ARDS } & \text { Acute Respiratory Distress Syndrome (akutes Lungenversagen) } \\ \text { ATLS } & \text { Advanced Trauma Life Support } \\ \text { BWS } & \text { Brustwirbelsäule } \\ \text { CCT } & \text { kraniale Computertomografie (Schädel-CT) } \\ \text { DGU } & \text { Deutsche Gesellschaft für Unfallchirurgie } \\ \text { ESIN } & \text { elastisch stabile intramedulläre Nagelung } \\ \text { FAST } & \text { Focused Assessment with Sonography for Trauma } \\ \text { GCS } & \text { Glasgow Coma Scale } \\ \text { HWS } & \text { Halswirbelsäule } \\ \text { ICP } & \text { Intracranial Pressure } \\ \text { ICR } & \text { Interkostalraum } \\ \text { ISS } & \text { Injury Severity Score } \\ \text { K-Draht } & \text { Kirschner-Draht } \\ \text { LiLa e.V. } & \text { Licht und Lachen für kranke Kinder - Effizienz in der Medizin e.V. } \\ \text { LWS } & \text { Lendenwirbelsäule } \\ \text { MSCT } & \text { Mehrschicht-Computertomografie } \\ \text { PCCF } & \text { pediatric comprehensive Classification of Long Bone Fractures } \\ \text { SCIWORA } & \text { Spinal Cord Injury without radiographic Abnormality } \\ \text { SHT } & \text { Schädel-Hirn-Trauma } \\ \text { VAC } & \text { Vacuum-assisted Closure } \\ & \end{array}$


Als Verletzungsursache bei Säuglingen und Kleinkindern überwiegen Stürze aus unterschiedlicher Höhe. In dieser Altersgruppe tritt auch der überwiegende Anteil an isolierten, geschlossenen Schädel-Hirn-Traumata auf.

In hohem Maß sind Säuglinge neben Unfällen durch tätliche Gewalt gefährdet und haben im Vergleich zu allen Kindern unter 15 Jahren das höchste Risiko, durch Unfälle oder Gewalteinwirkung zu sterben. Im Jahr 2012 war nach Angaben des Statistischen Bundesamtes die Rate der Verletzungen durch Gewalt sogar größer als die der Unfälle. Dabei muss von einer hohen Dunkelziffer ausgegangen werden.

Ab dem Schulkindalter nimmt die Inzidenz von Verkehrsunfällen deutlich zu, da die Kinder als Fußgänger, Fahrrad- oder später als Mofa- oder Mopedfahrer zunehmend aktiv am Verkehr teilnehmen. Jungen verunfallen nun auch häufiger als Mädchen (56,2\% Jungen im Jahr 2012), was mit der stärkeren Verkehrsbeteiligung und der größeren Risikobereitschaft zusammenhängt.

- Insgesamt gesehen ist der Verkehrsunfall der mit Abstand häufigste Unfallmechanismus.

Neben den o.g. Unfallmechanismen sind Kinder aller Altersstufen als Pkw-Beifahrer in Verkehrsunfälle verwickelt. Mit 7 getöteten Kindern je 1 Million Einwohner ist das Risiko, im Kindesalter (unter 15 Jahren) an einem Verkehrsunfall zu versterben, noch relativ gering; trotzdem starben im Jahr 201413 Kinder mehr als im Vorjahr (71 Kinder vs. 58 im Jahr 2013).

Eine weitere Verletzungsursache ab dem 12. Lebensjahr sind Suizidversuche und Suizide, die ab jetzt konstant für 5,1\% der Todesfälle ursächlich sind.

\section{Anatomische Grundlagen und Verletzungsformen}

Die Verletzungsmuster werden beim Kind durch die in der jeweiligen Altersstufe unterschiedlichen Körpergrößen und -proportionen sowie die unterschiedlichen Stadien der Skelettentwicklung bestimmt. Im diesem Abschnitt soll auf die anatomischen Besonderheiten der einzelnen Körperregionen eingegangen und daraus resultierende Verletzungsformen herausgearbeitet werden.

\section{Schädel}

Das Schädel-Hirn-Trauma ist die häufigste Todesursache im Kindesalter.
Bei Säuglingen und Kleinkindern kommt es infolge von Stürzen häufig zu Verletzungen des Schädels. Durch das ungünstige Kopf-Körper-Verhältnis mit dem in Relation zum Rumpf großen und schweren Kopf gepaart mit noch nicht ausgebildeten Schutzmechanismen und einer schwachen Nackenmuskulatur können bereits geringfügige Verletzungsmechanismen zu schweren SchädelHirn-Traumata führen.

Die beim Kind noch dünne Schädeldecke bietet wenig Schutz für das Gehirn, wodurch primäre intrazerebrale Verletzungen begünstigt werden. Die fehlende Spongiosa der Schädeldecke und die feste Verbindung zwischen Dura mater und Knochen erklären die speziellen Frakturformen (häufiger Impressionsfrakturen) und das seltene Auftreten von Epiduralhämatomen bei Kindern unter 3 Jahren. Der noch weiche Knochen bricht nicht scharfkantig und führt daher nur selten zu einer Verletzung der A. meningea media, die darüber hinaus noch nicht in einem knöchernen Kanal verläuft. Hingegen können im Säuglingsalter bereits geringfügige Gewalteinwirkungen - wie z.B. der Sturz vom Wickeltisch durch die Verformbarkeit des Schädels zu einem Abriss einer Brückenvene führen und somit ein subdurales Hämatom zur Folge haben.

Nach Schluss der Fontanellen zeigt das kindliche Gehirn eine geringe Compliance. Die noch offenen Suturen ermöglichen zwar eine geringe Volumenvergrößerung. Da die Blut-Hirn-Schranke beim Kind aber noch nicht voll ausgereift ist, zeigt das Gehirn eine hohe Bereitschaft zur Ödembildung, sodass bei fast einem Drittel der SchädelHirn-Traumata diffuse Hirnödeme auftreten.

Diffuse axonale Traumata entstehen in erster Linie durch axiale Gewalteinwirkung. Hierbei sind in der CT meist keine größeren parenchymatösen Läsionen nachweisbar,

\section{Definition}

\section{Shaken-Baby-Syndrom}

Das sog. Shaken-Baby-Syndrom bei Kindesmisshandlung wird dadurch verursacht, dass der schwere Kopf mit der oberen HWS nur eine geringe Resistenz gegen Torsionskräfte aufweist und es somit, neben den Akzelerationsund Dezelerationskräften, die auf den Schädel einwirken und zu einem diffusen Axonschaden führen, zu Quetschungen des zervikomedullären Übergangs mit entsprechender Hirnödembildung sowie zu subduralen Hämatomen und retinalen Blutungen kommt. 
die Kinder fallen jedoch durch einen primär schlechten GCS-Score auf.

Cave: Kinder mit Schädel-Hirn-Traumata zeigen eine ausgeprägte Neigung zur Hypoxie mit konsekutiver Ischämie, die wiederum zerebrale Infarkte mit diffuser Hirnschwellung auslösen kann.

\section{Wirbelsäule}

Insgesamt sind Verletzungen der Wirbelsäule aufgrund der Elastizität des Knochens und der geringen Masse des kindlichen Körpers selten. Nur 1-2\% aller verletzten Kinder weisen eine Wirbelsäulenverletzung auf. Beim schwer verletzten Kind bis zu einem Alter von 10 Jahren ist die Wirbelsäule in $8 \%$ der Fälle betroffen, der Anteil steigt auf $20 \%$ in der Gruppe der 11 - bis 15 -Jährigen.

Die häufigsten Wirbelsäulenverletzungen im Kindesalter finden sich im thorakolumbalen Bereich. Bei Kleinkindern überwiegen Verletzungen im mittleren thorakalen Bereich, bei Adoleszenten ist der thorakolumbale Übergang am häufigsten betroffen. Bei den Verletzungen der thorakalen und lumbalen Wirbelsäule bei Kindern handelt es sich nur selten um schwere Verletzungen.

Der Verletzungsmechanismus ist eine Kompression mit/ ohne Flexion, ebenso sind eine Distraktion sowie eine Scherung/Rotation (z.B. durch den Bauchgurt bei einem Pkw-Unfall) möglich. Häufigste Ursache für Verletzungen im BWS- und LWS-Bereich sind Verkehrsunfälle.

Je jünger das Kind hingegen ist, desto häufiger finden sich Verletzungen der Halswirbelsäule. Im Alter unter 9 Jahren machen HWS-Verletzungen die häufigsten Wirbelsäulenläsionen aus. Eine Besonderheit im Kleinkindalter sind ligamentäre Verletzungen am kraniozervikalen Übergang. Die noch horizontalen und flachen Gelenkfacetten werden von einem schwachen Muskelapparat mit laxen Bandstrukturen bei großer KopfKörper-Relation gehalten. Daher entsteht bei Krafteinwirkung eine hohe Belastung.

Etwa 70\% aller kindlichen HWS-Läsionen betreffen Atlas und Axis, wobei komplexe Verletzungen ein hohes Letalitätsrisiko aufweisen. Beim Kleinkind artikuliert der Kopf mit dem Atlas horizontal, wobei die straffe Führung der Articulatio atlantooccipitalis vorwiegend die Flexion/ Extension, aber wenig Rotation und Seitneigung zulässt.

Die kindlichen Bandstrukturen der Articulationes atlantoaxiales (kombiniertes Drehgelenk) lassen eine Rotation von ca. $20-60^{\circ} \mathrm{zu}$, wodurch Bandrupturen und Verlet-

\section{Hintergrund}

\section{SCIWORA}

Da die kindliche Wirbelsäule im Vergleich zur Erwachsenenwirbelsäule ein deutlich höheres Elastizitätsmodul aufweist, sind Dehnungen des Achsenskeletts in vertikaler Richtung bis zu $5 \mathrm{~cm}$ möglich, ohne dass Verletzungen an der Wirbelsäule resultieren. Das Rückenmark hingegen toleriert nur eine longitudinale Dehnung von ca. $0,5 \mathrm{~cm}$, bis es zum Zerreißen oder schwerwiegenden strukturellen Defekten kommt. Hierdurch lassen sich das Auftreten eines SCIWORA-Syndroms (spinal Cord Injury without radiographic Abnormality) sowie multisegmentale neurologische Störungen erklären.

Durch die zunehmende Optimierung der MRT-Diagnostik werden jedoch heutzutage in der Regel morphologische Befunde festgestellt, die mit den neurologischen Ausfällen korrelieren.

zungen der Facettengelenke mit Subluxation möglich werden.

- Eine Subluxationsstellung bis 2-3 mm an der oberen HWS ist bei Kindern physiologisch!

Mit dem Wachstum vergrößern sich die Neigungsebenen der Gelenkflächen bei $\mathrm{C} 1 / \mathrm{C} 2$ von 55 auf $70^{\circ}$ und bei $\mathrm{C} 2-\mathrm{C} 4$ von 30 auf $60-70^{\circ}$ mit einer daraus folgenden höheren knöchernen Stabilität.

Die Wirbelsäule reift ab dem 8.-10. Lebensjahr mit stabileren Bändern und Gelenken, steileren Gelenkfacetten und rechteckigen Wirbelkörperformen aus und nähert sich anatomisch der Erwachsenenwirbelsäule.

Demzufolge ähneln ab dem Lebensalter von 8-10 Lebensjahren sowohl die Verletzungs- als auch Heilungsvorgänge denen bei Erwachsenen.

\section{Thorax und Abdomen}

Bei Verletzungen des Thorax und Abdomens stehen in Deutschland stumpfe Unfallursachen im Vordergrund. Der kindliche Brustkorb weist aufgrund der noch elastischen knöchernen Strukturen im Vergleich zum Erwachsenen eine erhöhte Compliance auf. Das führt dazu, dass von außen einwirkende Kräfte verstärkt nach intrathorakal übertragen werden, was die Entstehung innerer Verletzungen begünstigt, während Rippenserienfrakturen eher eine Seltenheit sind. 
Die Schockwellen im Rahmen eines Unfalls führen zu Kompressions- und Scherkräften mit Gewebezerreißungen. Häufigste Unfallfolgen sind somit

- die Lungenkontusion,

- der Pneumothorax und

- der Hämatothorax.

Verletzungen von Trachea, Bronchien, zentralen Gefäßen, Herz oder Zwerchfell treten beim Kind kaum auf. Pathophysiologisch entstehen Lungenkontusionen durch Einwirkung von Scherkräften auf die Lunge, die zur Zerreißung von Parenchymanteilen führen. Als Folge entstehen Leckagen kleinerer Gefäße mit Austritt von Blut in die Alveolen oder Blut und/oder Luft in den Pleuraspalt. Atelektasen, interstitielle Ödeme und proinflammatorische Reaktionen führen zu einem gestörten VentilationsPerfusions-Verhältnis mit drohender Hypoxie.

Die Oberbauchorgane sind bei Kindern verhältnismäßig größer als bei Erwachsenen, gleichzeitig steht das Zwerchfell tiefer. Der kleine, elastische Brustkorb sowie die noch gering entwickelte Muskulatur können Leber und Milz nur unzureichend Schutz bieten, was eine höhere Gefährdung für eine relevante intraabdominelle Verletzung auch bei nicht so hoher Gewalteinwirkung bedeutet. Verletzungen dieser Organe treten somit, ebenso wie Darmverletzungen, bei polytraumatisierten Kindern häufig auf. Zwerchfellrupturen treten selten auf ( $<1 \%$ der Fälle), dienen jedoch als Marker der Verletzungsschwere und sind häufig mit einem letalen Ausgang vergesellschaftet.

\section{Becken}

Das Becken entwickelt sich aus den primären Wachstumszentren Darm-, Sitz- und Schambein. Sie verbinden sich im Bereich der Hüftpfanne über die Y-Fuge, die sich im Alter von 12-15 Jahren verschließt. Die sekundären Wachstumszentren bilden die Apophysen, die im Alter zwischen 16 und 25 Jahren knöchern mit dem Becken fusionieren.

Das kindliche Becken unterscheidet sich von dem des Erwachsenen durch die großen knorpeligen Flächen und die höhere Elastizität auch der knöchernen Anteile. Daher können auf das kindliche Becken größere energetische Kräfte einwirken, ohne Frakturen auszulösen.

Beckenfrakturen sind somit ein Ausdruck besonders hoher Gewalteinwirkung beim Kind und trotz oft „einfach" imponierender Frakturform mit einem höheren Risiko von begleitenden Organverletzungen vergesellschaftet.
Auch bei gering dislozierten Frakturen müssen Begleitverletzungen des Urogenitaltrakts und des Rektums ausgeschlossen werden, zumal das insgesamt flachere Becken des Kindes weniger Schutz bieten kann. Verletzungen der Wachstumszonen, insbesondere der Y-Fuge, können mit Wachstumsstörungen bis hin zur vollständigen Hypoplasie einer Beckenhälfte einhergehen. Vordere Beckenringverletzungen entstehen durch direkten Anprall. Häufiger als Beckenringfrakturen treten beim Kind Beckenrandfrakturen auf. Über 90\% dieser Frakturen sind stabil und bedürfen keiner operativen Therapie.

Azetabulumfrakturen kommen beim Kind extrem selten vor. Ihr Anteil liegt bei 0,8-15\% aller Beckenfrakturen im Kindesalter. Die Azetabulumfraktur im Kindesalter entsteht im Gegensatz zur Beckenfraktur meist durch einen indirekten Anprall. Auch hier können Wachstumsstörungen resultieren. Je jünger das Kind, desto schwieriger ist die Diagnostik.

Eine besondere Frakturform im Wachstumsalter sind die sog. Avulsionsfrakturen in den Apophysenregionen, die Ausdruck der Unreife des Knorpels und offener Wachstumsfugen sind.

\section{Extremitäten}

Grundsätzlich können Kinder aufgrund der höheren Plastizität und Elastizität des Knochens und der relativ dicken Kortikalis Deformierungen auch im Bereich der Extremitäten partiell abfangen, ohne dass Frakturen entstehen. Kommt es zu einer Frakturierung, so findet sich diese überwiegend im diaphysären Bereich und nur selten epi-/metaphysär, was am ehesten durch den hohen knorpeligen Anteil in diesem Bereich erklärt werden kann.

\section{Klassifikationen}

\section{Schädel-Hirn-Trauma}

- Bei Schädel-Hirn-Traumata ist die Glasgow Coma Scale (GCS) internationaler Standard in der Beurteilung neurologischer Defizite.

Beurteilt und mit einer abgestuften Punktzahl bewertet wird die Reaktion der Augen sowie des motorischen und verbalen Systems auf Ansprache.

Ab einer Punktzahl von $<12$ muss von einem schweren Schädel-Hirn-Trauma (SHT) ausgegangen werden. Bei 
einem Score-Wert von $\leq 8$ wird der Patient als komatös mit entsprechend ernster Prognose eingestuft.

Prognostische Aussagen lassen sich aus der GCS nicht ableiten. Eingeschränkt ist die Beurteilung von Säuglingen und Kleinkindern anhand der üblichen verwendeten GCS. Da sich jedoch eine speziell für pädiatrische Patienten entwickelte GCS nicht allgemein durchsetzen konnte, erfolgt heute meist auch bei Säuglingen und Kleinkindern in Anlehnung an die für Erwachsene entwickelte GCS eine subjektive Einschätzung durch den erstbehandelnden Arzt.

\section{Polytrauma}

Zur Klassifizierung der Verletzungsschwere bei polytraumatisierten Patienten wurde eine Vielzahl von anatomisch-morphologischen und physiologischen Scores entwickelt, hierunter auch einige, die speziell für pädiatrische Patienten erarbeitet wurden. Durchgesetzt hat sich klinisch jedoch sowohl zur Beurteilung der Verletzungsschwere bei erwachsenen als auch bei kindlichen Polytraumata der von Baker entwickelte Injury Severity Score (ISS). Der ISS bewertet die Schwere der Verletzungen der Körperregionen mit Punkten, die nach der Abbreviated Injury Scale (AIS) festgelegt werden. Aus der Summe der Quadrate der bei der AIS ermittelten 3 höchsten Punktwerte wird dann der ISS berechnet.

Studien belegen, dass eine direkte Beziehung zwischen ermitteltem ISS und Letalität, Morbidität, späterer Invalidität sowie der Dauer der Intensiv- und Krankenhausbehandlung bei polytraumatisierten Patienten besteht.

\section{Wirbelsäulenverletzungen}

Wirbelsäulenverletzungen bei Kindern über 8 Jahren zeigen alle Verletzungsformen, die auch bei Erwachsenen auftreten können. Sie werden daher klassisch in Kompressions-, Flexions-Distraktions- und Rotationsverletzungen nach der AO-Klassifikation (Magerl et al. [1]) eingeteilt.

Bei Kindern unter 8 Jahren finden sich Verletzungen der knorpeligen Anlagen (Frakturen der knorpeligen Wirbelkörperendplatten und Wachstumsfugen). Hier erfolgt eine Einteilung nach Blauth [2]:

- Lösung der unteren knorpeligen Wirbelkörperendplatte (Salter-Harris-I-Läsion),

- Abbruch der vorderen unteren Wirbelkörperkante (Aitken-II-, Salter-Harris-III-Fraktur).

\section{Beckenfrakturen}

Bei Beckenfrakturen unterscheiden die Klassifikationen grundlegend entweder zwischen

- stabilen und instabilen Frakturen oder

- Frakturen, die ohne Folgen ausheilen, gegenüber solchen, die mit Defekt ausheilen.

Auch im Kindesalter werden Beckenverletzungen vorwiegend nach den Klassifikationssystemen der Erwachsenenchirurgie eingeteilt. Ab dem 14. Lebensjahr (Verschluss der Y-Fuge) entsprechen die Frakturformen denen des Erwachsenen.

Beckenringfrakturen werden nach der üblichen AO-Klassifikation (Pennal et al. $[3,4]$ ) eingeteilt (Abb. 1) in

- stabile A-,

- partiell (rotatorisch) instabile B- und

- (vertikal) instabile C-Verletzungen.
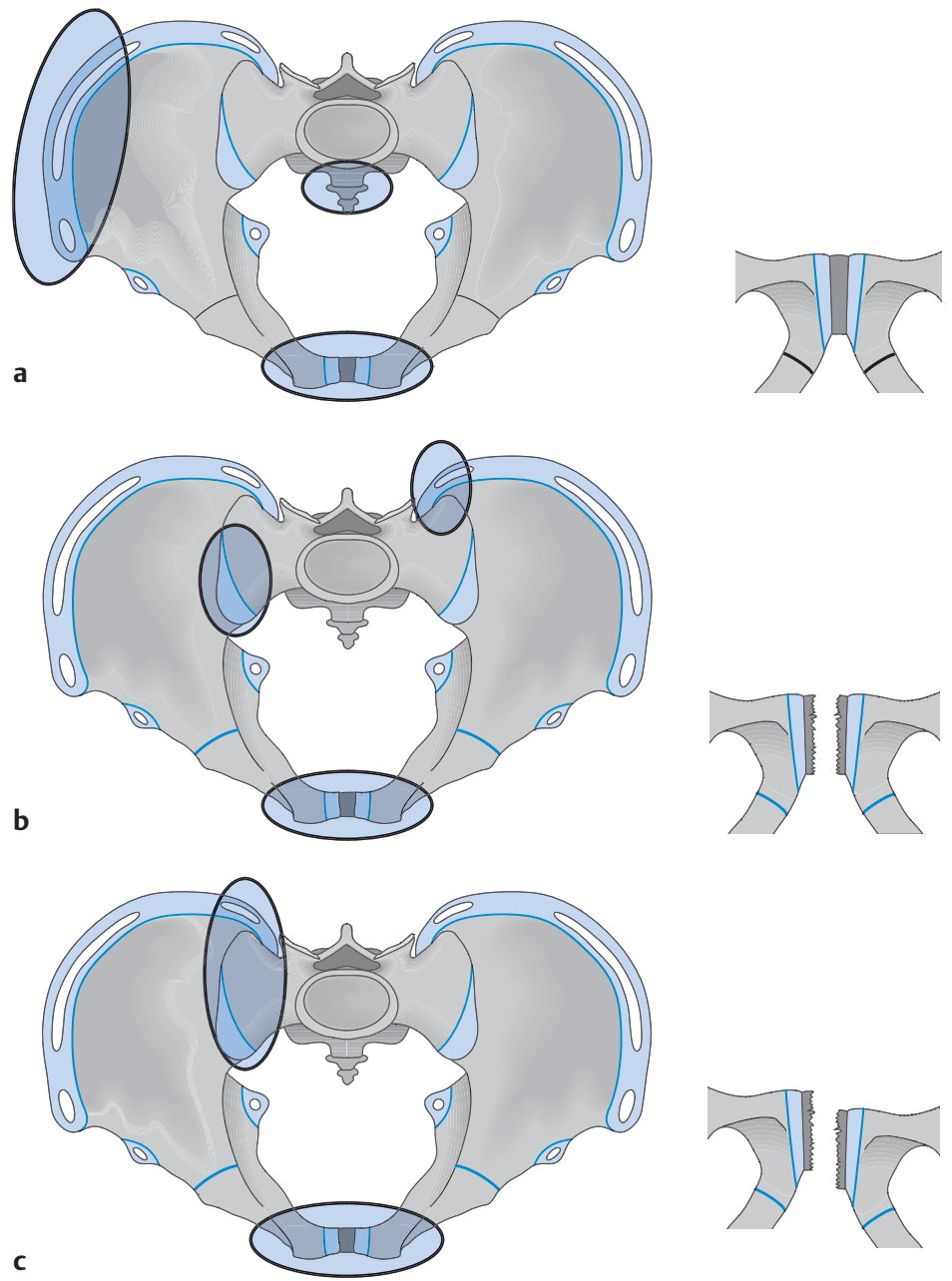

Abb. 1 - Klassifikation der Beckenringfrakturen nach Pennal u. Tile [3]. a Stabile A-Verletzungen. b Partiell (rotatorisch) instabile B-Verletzungen. c (Vertikal) instabile C-Verletzungen. 


\section{Extremitäten}

Extremitätenfrakturen im Kindesalter werden im klinischen Alltag entsprechend ihrer Beziehung zur Epiphysenfuge nach Salter und Harris (Aitken) $[5,6,7]$ klassifiziert. Übergangsfrakturen im Jugendalter werden gesondert eingeteilt.

In den letzten Jahren wurden, basierend auf der AOKlassifikation für Erwachsene, parallel 2 umfassende Dokumentationssysteme entwickelt, die alle Besonderheiten kindlicher Frakturen berücksichtigen, deren Anwendung im klinischen Alltag sich jedoch noch nicht durchsetzen konnte. Es handelt sich einerseits um die „AO-Klassifikation für Frakturen im Wachstumsalter“ ( $\mathrm{PCCF}=$ pediatric comprehensive Classification of Long Bone Fractures) [8,9], andererseits um die „LiLa-Klassifikation für Frakturen der langen Röhrenknochen im Kindesalter“. Letztere wurde von dem gemeinnützigen Verein LiLa e.V. entwickelt [10].

\section{Diagnostisches Vorgehen}

\section{Anamnese, Bewusstseinsprüfung}

Anamnestische Angaben sind oft ungenau und lassen nur in Einzelfällen auf die Art der Verletzung schließen. Die Bedeutung der Anamnese besteht daher beim Kind vor allem in der Erfassung der Schmerzlokalisation und -intensität. Zudem sollten insbesondere bei SchädelHirn-Traumata die Vigilanz und Reaktion des Kindes mitbeurteilt werden. Das Auftreten und die Intensität von vegetativen Störungen sollte ebenfalls in die Beurteilung der Schwere der Verletzung mit einfließen.

\section{Klinik}

Im Rahmen der Diagnostik sollten unnötige schmerzhafte Manipulationen weitgehend vermieden werden.

Bei Verdacht auf Frakturen im Körperstamm- oder Extremitätenbereich muss die Überprüfung der lokalen Druckschmerzhaftigkeit auf das notwendige Minimum beschränkt werden. Eine Beurteilung der Peripherie hinsichtlich Durchblutung, Motorik und Sensibilität gehört jedoch in jedem Fall zur erforderlichen Basisuntersuchung und muss auch entsprechend dokumentiert werden.

\section{Schockraummanagement}

Handelt es sich um ein schwer verletztes oder gar polytraumatisiertes Kind, sollte eine standardisierte Schockraumdiagnostik erfolgen, um in möglichst kurzer Zeit unter Sicherung der Vitalfunktionen das Gesamtverletzungsmuster zu erfassen und eine differenzierte Behandlung einleiten zu können. Hier empfiehlt sich ein Schockraummanagement in Anlehnung an das für Erwachsene entwickelte ATLS-Konzept (ATLS = Advanced Trauma Life Support) nach eingeübten Algorithmen und festgelegten Standards.

Das polytraumatisierte Kind sollte somit bei Eintreffen in der Klinik von einem möglichst eingespielten Traumateam im Schockraum empfangen werden. Das Team sollte neben dem koordinierenden Traumatologen, dem Anästhesisten sowie dem Radiologen ärztlicherseits durch einen pädiatrisch erfahrenen Kollegen (Pädiater oder pädiatrischen Intensivmediziner), optimalerweise ergänzt durch einen Kinderchirurgen oder in Kinderchirurgie erfahrenen Chirurgen ergänzt werden. In manchen Kliniken wird der unfallchirurgische Part durch die Kinderchirurgen übernommen. Weiterhin sollte in Abhängigkeit vom Verletzungsmuster das Team kurzfristig erweiterbar sein, z. B. durch einen Neurochirurgen, MundKiefer-Gesichts-Chirurgen, Augenheilkundler etc.

Anästhesist und Pädiater sind verantwortlich für die Beatmung, das Monitoring, die Volumenersatztherapie und die Blutentnahmen. Der Unfallchirurg führt parallel zu diesen Maßnahmen unter Berücksichtigung der Anamnese die körperliche Untersuchung sowie die evtl. erforderlichen chirurgischen Sofortmaßnahmen durch (z.B. Anlage von Thoraxdrainagen, Blutstillung, Repositionen usw.). Hierbei werden auch die Art und der Umfang der bildgebenden Akutdiagnostik sowie die Prioritätenliste der weiteren Behandlungsabfolge in Abhängigkeit vom klinischen Gesamtbild vom Traumatologen festgelegt.

Eine standardisierte Schockraumversorgung mit klar festgelegtem Polytraumaalgorithmus hilft, Sekundärschäden zu vermeiden, die Zeit nicht aus den Augen zu verlieren und eine gleichbleibende Qualität der Versorgung zu gewährleisten. 


\section{Bildgebende Verfahren}

\section{[ Röntgendiagnostik}

Beim polytraumatisierten Kind gehört ebenso wie beim Erwachsenen eine a.-p. Röntgenaufnahme des Thorax und bei entsprechendem Unfallmechanismus eine Beckenübersichtsaufnahme zur Standarduntersuchung im Rahmen der Schockraumdiagnostik. Beim Säugling und Kleinkind kann hierzu eine Übersichtsaufnahme auf einer Röntgenplatte erfolgen.

Extremitäten werden zum Nachweis einer Fraktur in 2 Ebenen geröntgt. Die Röntgenaufnahme erfolgt prinzipiell mit dem/den angrenzenden Gelenk(en), sodass komplette osteochondrale Einheiten abgebildet werden.

\section{- Sonografie}

Die Sonografie nimmt in der Schockraumdiagnostik zur Erfassung intraabdomineller und thorakaler freier Flüssigkeit eine zentrale Stellung ein. Sie ist im Rahmen der Primärdiagnostik in der FAST-Technik (FAST = focused Assessment with Sonography for Trauma) obligat.

Mit der Sonografie lassen sich nicht nur freie Flüssigkeitsmengen, sondern auch entsprechende Läsionen an Leber und Milz nachweisen. Darüber hinaus eignet sich die Sonografie zum Nachweis von Pleura- und Perikardergüssen. Nachteil ist die geringe Sensitivität von 56,5\% zum Nachweis einer intraabdominellen Verletzung, sodass eine negative Sonografie diese nicht ausschließt und weitere Indikatoren in die Untersuchung einbezogen werden müssen.

\section{- Computertomografie}

Beim Erwachsenen konnte gezeigt werden, dass die routinemäßige Durchführung einer Ganzkörper-CT im Rahmen der Schockraumdiagnostik die Letalität senkt. Bei Kindern wird aufgrund der möglichen Spätfolgen durch die hohe Strahlenexposition der Einsatz in den letzten Jahren sehr kontrovers diskutiert. Um eine unnötige Strahlenbelastung zu vermeiden, ist die Indikation für eine CT-Untersuchung beim Kind individuell zu prüfen und eine Risiko-Nutzen-Abwägung durchzuführen. Keinesfalls sollten dabei relevante Verletzungen übersehen werden oder eine entscheidende Zeitverzögerung in Kauf genommen werden.

Bei einem entsprechenden Unfallmechanismus mit dem Verdacht auf eine schwere Mehrfachverletzung erscheint die Durchführung der CT gerechtfertigt, um eine sichere Diagnostik zu erzielen und Verzögerungen zu vermeiden.

Eine klare Indikation für die Durchführung einer CT ergibt sich auch beim intubierten und neurologisch nicht beurteilbaren Kind. Sie wird heutzutage nach etablierten Kinderprotokollen unter Berücksichtigung von Größe und Gewicht möglichst strahlungsarm als kontrastmittelgestützte Mehrschicht-CT (MSCT) des Schädels (nativ), Thorax, Abdomens und Beckens durchgeführt (sog. Traumascan, Traumaspirale). Durch Anwendung von Niedrigdosisprogrammen soll laut Studien die Strahlenbelastung in den nächsten Jahren weiter deutlich reduziert werden können.

\section{- Magnetresonanztomografie}

Die Kernspintomografie spielt im Rahmen der Akutdiagnostik nur eine untergeordnete Rolle. In der Traumatologie liegt ihr Schwerpunkt in der Diagnostik von Wirbelsäulenverletzungen ohne primär erkennbare knöcherne Verletzungen.

> Bei neurologischen Ausfällen muss eine MRT der Wirbelsäule in Höhe der vermuteten Läsion durchgeführt werden, wenn in der CT keine knöcherne Läsion aufgedeckt werden kann.

Hauptvorteil ist die fehlende Strahlenbelastung. Dennoch sollte die Indikation, insbesondere bei Kleinkindern, kritisch gestellt werden, da diese meist aufgrund der langen Untersuchungszeit sediert werden müssen.

\section{Therapeutisches Vorgehen}

\section{Schädel-Hirn-Trauma}

Bei Kindern mit einer initialen GCS von 15 Punkten besteht zunächst keine Indikation zur Durchführung einer primären computertomografischen Untersuchung, wenn eine initiale Bewusstlosigkeit ausgeschlossen werden kann und ein unauffälliger neurologischer Untersuchungsbefund vorliegt. Bei einer fraglichen initialen Bewusstlosigkeit, ausgeprägter vegetativer Begleitsymptomatik (Übelkeit, Erbrechen, Schwindel) oder bei nicht sicher einschätzbarer Vigilanz des Kindes sollte jedoch eine stationäre Aufnahme des Kindes zur Überwachung erfolgen. Bei Verschlechterung der Bewusstseinslage besteht dann sekundär die Indikation zur CCT. 


\section{Pädiatrische Orthopädie und Unfallchirurgie}

Übersicht

\section{Grundsätzlich zu beachten bei Schädel-Hirn-Trauma}

- Ein initialer GCS-Wert von < 14 Punkten ist eine Indikation zur gezielten stationären Überwachung.

- Bei infolge der Kopfverletzung somnolenten und entscheidend vigilanzgeminderten Kindern besteht die Indikation zur Frühintubation, um die Aspirationsgefahr zu vermindern und einen hypoxischen Hirnschaden zu vermeiden.

- Bei polytraumatisierten Kindern, die intubiert und beatmet in die Klinik eingeliefert werden, sollte im Rahmen der initialen Diagnostik immer ein CCT erfolgen.

- Nach Primärstabilisierung hat die Therapie eines operationspflichtigen SHT immer Vorrang, da das SHT nach stumpfen Verletzungen auch bei Kindern die Haupttodesursache darstellt.

- Bei raumfordernden, blutungsbedingten intrakraniellen Druckerhöhungen muss möglichst schnell eine operative Entlastung erfolgen.

- Sowohl für das epidurale als auch für das subdurale Hämatom muss ab der 3. Stunde nach Trauma mit einer signifikanten Verschlechterung des Behandlungsergebnisses gerechnet werden.

- Epiduralhämatome werden durch eine Trepanation über dem Hämatom entlastet. Die Prognose ist bei frühzeitiger Therapie gut, hängt jedoch vom Ausmaß der Hirnkompression ab.

\section{- Operative Versorgung}

Akute subdurale Hämatome werden durch eine große temporale Entlastungstrepanation mit Duraerweiterungsplastik entlastet. Durch die Duraerweiterungsplastik können Reserveräume geschaffen werden.

Bei isolierten intrazerebralen Läsionen, die essenzielle Hirnareale komprimieren, sollte bei Kindern frühzeitiger als beim Erwachsenen die Indikation zur operativen Entlastung gestellt werden.

D Die uni- bzw. bitemporale Entlastungskraniektomie hat sich in den letzten Jahren als pathophysiologisch sinnvollstes Konzept erwiesen, um schwellungsbedingte Sekundärschäden zu vermeiden. Sie sollte Studien zufolge bei Kindern nicht mehr als Ultima Ratio angesehen werden, sondern als Therapiekonzept.

Kinder mit hohem intrakraniellem Druck und Kompression des Mittelhirns zeigen im Vergleich zu ähnlich verletzten Erwachsenen bessere Ergebnisse.

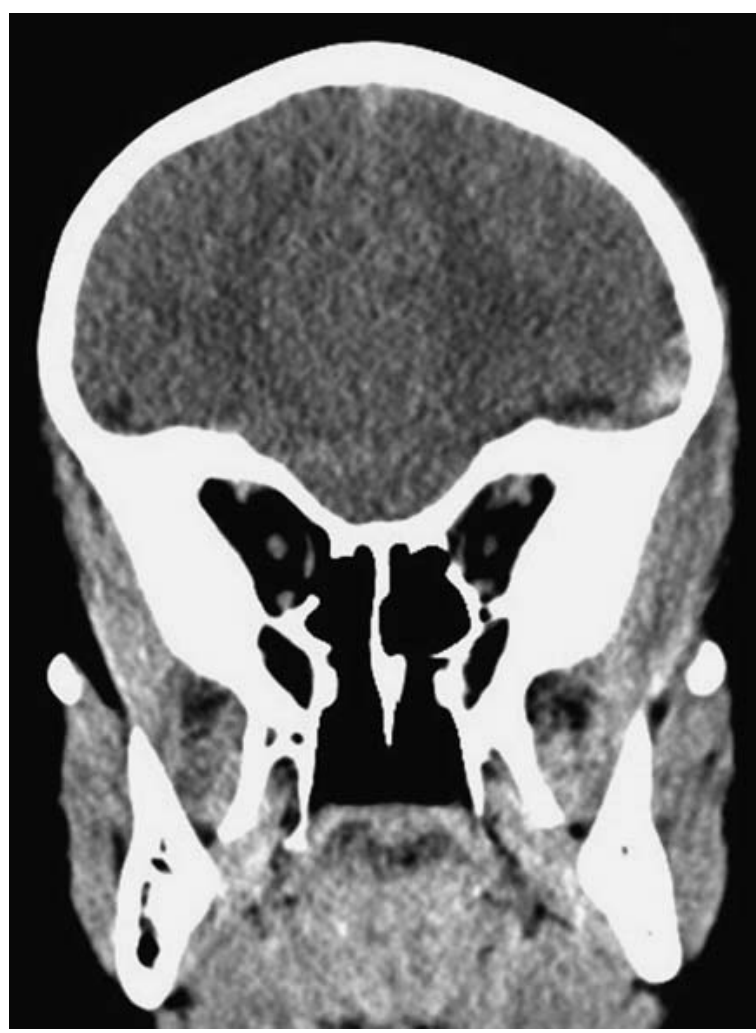

Abb. 2 - Schädel-CT (CCT) eines 6-jährigen Mädchens nach Sturz aus $4 \mathrm{~m}$ Höhe auf Beton mit Nachweis eines Epiduralhämatoms links frontotemporal.

Da gerade Kinder eine ausgeprägte Neigung zur diffusen Hirnschwellung mit konsekutiver Ischämie und Infarkten zeigen, sollte bei Vorliegen eines schweren SHT und einer GCS $<8$ bei geschlossenen Fontanellen eine intraparenchymatös lokalisierte Hirndrucksonde angelegt werden. Diese kann sowohl auf der traumadominanten als auch auf der kontralateralen Seite platziert werden. Essenziell ist ein intensives Monitoring mit aggressiver Hirndrucktherapie.

Klinische Beispiele sind in Abb. 2 u. Abb. 3 dargestellt.

- Cave: Aufgrund des Risikos der Entstehung eines Hirnödems und des Risikos eines Cerebral-Salt-wastingSyndroms ist beim polytraumatisierten Kind auf ein subtiles Flüssigkeitsmanagement zu achten.

\section{Thoraxtrauma}

Rippenfrakturen sind beim Kind selten. Ein Fehlen von Rippenfrakturen schließt intrathorakale Verletzungen, Hämato- oder Pneumothoraces nicht aus. 


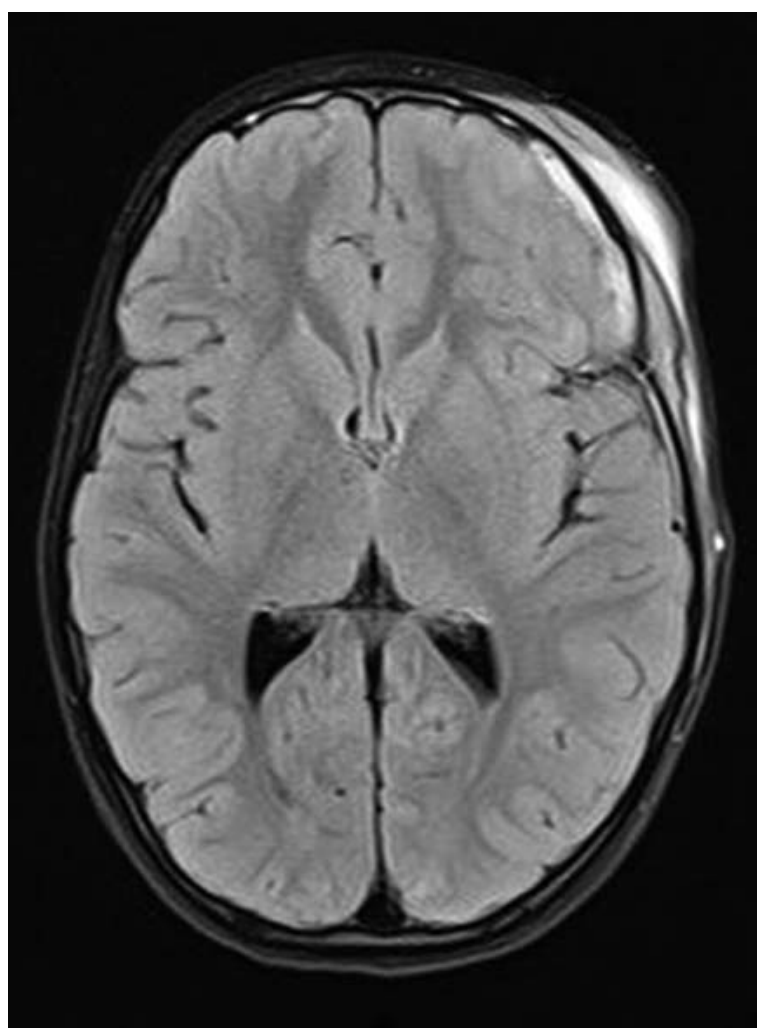

Abb. 3 - Nachweis einer subarachnoidalen Blutung links frontotemporal basal sowie einer nicht dislozierten Schädelkalottenfraktur linksseitig frontotemporal (nicht mit abgebildet). Nach initialer Reanimationspflicht bei GCS 9 war keine Intubation erfolgt. Im Schockraum GCS 13. Auf eine Intervention bzw. die Anlage einer ICP-Sonde.

\section{- Thoraxdrainage}

Beim Pneumo- und/oder Hämatothorax besteht die Indikation zur Anlage einer Thoraxdrainage. Die Anlage der Thoraxdrainage erfolgt auch beim Kind über eine Minithorakotomie, die ein stumpfes Einbringen der Drainage unter digitaler Kontrolle ermöglicht (s. Infobox „OP-Schritte und Tricks“ u. Abb.4).

- Cave: Auch beim Kind Anlage der Thoraxdrainage nie unter Mamillenhöhe!

\section{- Operative Versorgung}

Indikationen zur operativen Versorgung bestehen beim kindlichen Thoraxtrauma eher selten. Mögliche Operationsindikationen sind:

- anhaltende kreislaufwirksame Blutungen aus der Thoraxdrainage,

- nach intrathorakal durchspießende Fremdkörper mit Verletzung des Lungenparenchyms,
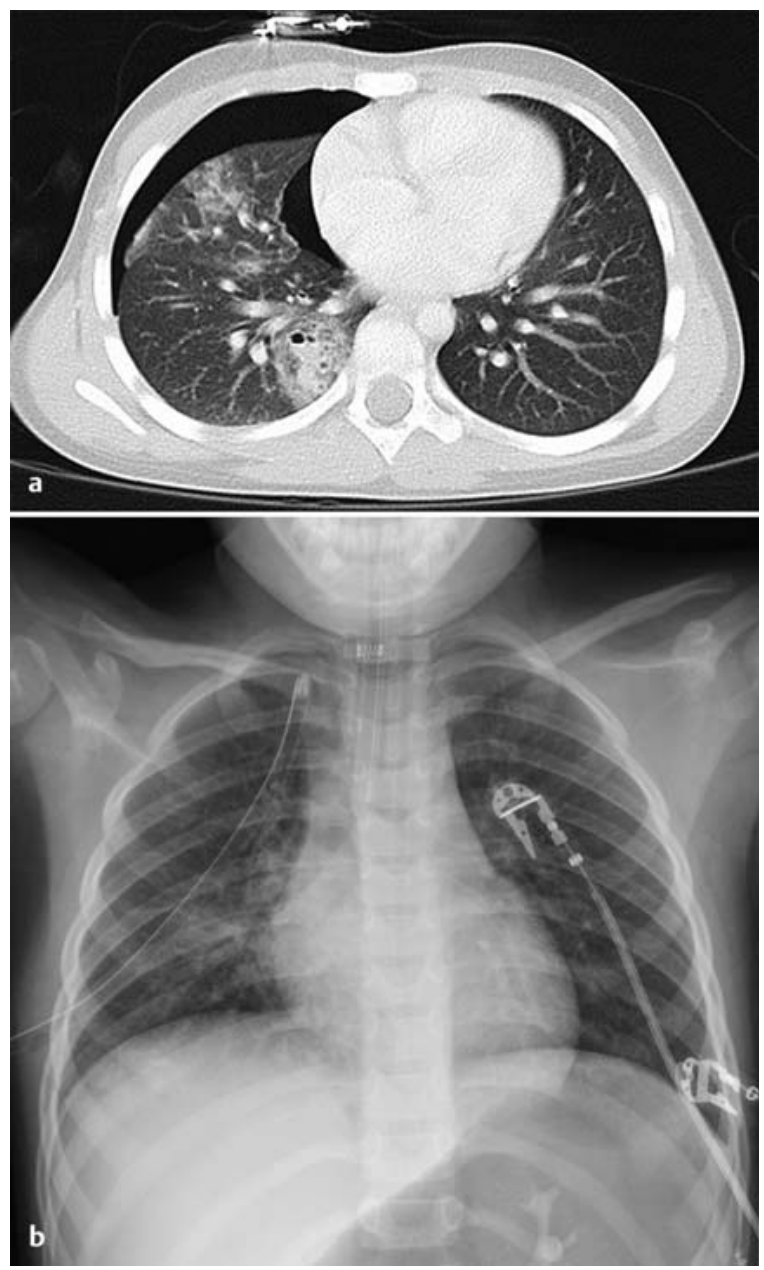

Abb. 4 - 6-jähriges Mädchen, das als Fahrradfahrerin mit dem Anhänger eines Pkw kollidiert war. a Initiales Thorax-CT: Es besteht ein ventraler Pneumothorax rechts und eine Kontusion im Bereich des Mittelund Unterlappens. b Thoraxröntgenbild nach Anlage einer BülauDrainage.

OP-Schritte und Tricks

\section{Anlagetechnik Thoraxdrainage}

\section{Thoraxdrainage durch Minithorakotomie}

- 3-4 cm lange Hautinzision in Höhe des 5. ICR in der mittleren Axillarlinie

- subkutane Präparation und Inzision der Interkostalmuskulatur am Oberrand der 6. Rippe

- Perforation der Pleura und digitale Exploration des Pleuraraums

- stumpfes Einführen der Thoraxdrainage bei zurückgezogenem Trokar über den Finger (Leitschiene) in die gewünschte Position

Monaldi-Drainage bei ventralem Pneumothorax

- Hautinzision in Höhe des 2. ICR in der Medioklavikularlinie

- Präparation und Eingehen am Oberrand der 3. Rippe 
- Perikardtamponade,

- tracheobronchiale Verletzungen, die nicht durch den Tubus abgedichtet werden können und ein persistierend intolerables Luftleck aufweisen.

Tracheobronchiale Verletzungen sind allerdings selten und werden meist erst bronchoskopisch diagnostiziert. Inkomplette Rupturen mit weniger als $1 / 4$ der Zirkumferenz können bei Abdichtung durch den Tubus bzw. tolerablem Luftleck unter wiederholter bronchoskopischer Kontrolle konservativ zur Ausheilung gebracht werden.

Am häufigsten werden beim Kind als Folge des Thoraxtraumas Lungenkontusionen beobachtet. Diese stehen aufgrund der möglichen daraus resultierenden respiratorischen Insuffizienz im Mittelpunkt der Behandlung. Atelektasen und interstitielle Ödeme führen zu Pneumonien und einem gestörten Ventilations-Perfusions-Verhältnis mit drohender Hypoxie. Es besteht somit die Indikation zur frühzeitigen Intubation und Respiratorbehandlung. Das Pneumonierisiko bei schweren Lungenkontusionen beträgt 20-30\%. Eine frühzeitige antibiotische Behandlung ist somit indiziert.

\section{Abdominaltrauma}

\section{- Konservative Therapie}

In den letzten Jahren ist die Behandlung stumpfer Abdominaltraumata mit Verletzung solider Organe wie Leber, Milz oder Nierenparenchym zunehmend zur Domäne der konservativen Therapie geworden.

Wie eine groß angelegte Studie aus Toronto zeigt, können Milzverletzungen bei Kindern in 99\% der Fälle erfolgreich konservativ behandelt werden. Zahlen aus europäischen Kliniken (Niederlande) zeigen, dass hier in 91\% eine konservative Behandlung möglich war und in $97 \%$ der Fälle zum Erfolg führte.

Sonografisch nachweisbare größere Flüssigkeitsmengen sowie computertomografisch nachweisbare höhergradige Verletzungen von Leber, Milz und Nierenparenchym werden bei Kindern toleriert und ein primär konservatives Vorgehen angestrebt (Abb. 5). Essenziell sind allerdings eine engmaschige intensivmedizinische Überwachung und bei akuter Verschlechterung der Kreislaufverhältnisse eine sofortige OP-Bereitschaft.

Bezüglich der Leberverletzungen sind die Zahlen ähnlich. In Studien konnte gezeigt werden, dass selbst Leberverletzungen Grad 4 und 5 nach Moore folgenlos unter konservativer Therapie ausheilen können.

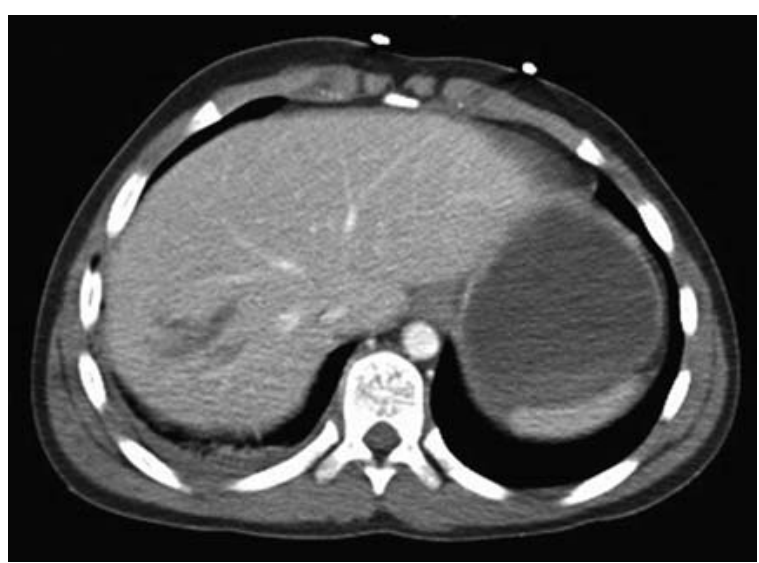

Abb. 5 - Abdomen-CT eines 12-jährigen Jungen, der als Fußgänger von einem Motorradfahrer angefahren und mitgeschleift worden war. Es zeigt sich eine Leberkontusion/-lazeration im Bereich der Segmente 7 und 8 mit wenig perihepatischer Flüssigkeit, die konservativ behandelt wurde.

Cave: Hauptproblem der konservativen Behandlung ist das Übersehen von Darmverletzungen.

\section{- Operative Versorgung}

Besteht trotz Volumensubstitution und Transfusion eine persistierende Kreislaufinstabilität bei gleichzeitig sonografisch nachweisbarer freier Flüssigkeit intraabdominell, so sollten die Kinder ohne vorausgegangene CT-Diagnostik und ohne Zeitverzögerung einer operativen Therapie zugeführt werden.

Cave: Kinder reagieren erst sehr spät mit Blutdruckabfall auf anhaltende Blutungen. Eine manifeste Tachykardie bei gleichzeitig kalter Peripherie muss daher als Schockzeichen mit drohender Dekompensation gewertet werden.

Wenn möglich, sollten bereits bei der primären operativen Versorgung sämtliche Läsionen endgültig versorgt werden.

Beim Kind sollte sowohl bei Milz- als auch bei Leberverletzungen ein Organerhalt angestrebt werden.

Handelt es sich um eine massive, chirurgisch nicht stillbare Blutung aus dem Leberparenchym, ist wie beim Erwachsenen das perihepatische Packing das primäre Mittel der Wahl. Sollte die Schockraumdiagnostik zugunsten der operativen Versorgung der abdominalen Verletzung unterbrochen worden sein, sollte diese nach operativer Versorgung des Abdomens komplettiert werden. War ein Packing erforderlich, wird nach Kreislauf- 
Indikationen

\section{Indikationen zur primär operativen Therapie abdominaler Verletzungen}

- kreislaufinstabile Patienten mit sonografisch nachweisbarer freier Flüssigkeit intraabdominell

- penetrierende Verletzungen

- schwere extraabdominale Zusatzverletzungen, wie z. B. Beckenfrakturen nach Überrolltrauma

- schwere tiefe Läsionen von Leber und Milz mit hoher Wahrscheinlichkeit von Hohlorganverletzungen

- Hohlorganverletzungen

- Blasenverletzungen

- Zwerchfellruptur
Studienlage mit einem besseren Verlauf im Vergleich zum konservativen Vorgehen einhergehen.

Besteht im primären CT der Verdacht auf eine Zwerchfellruptur, ist auch hier die Indikation zum primär operativen Vorgehen gegeben. Bei Zwerchfellrupturen bestehen meist intraabdominelle Begleitverletzungen, sodass die Versorgung über eine Laparotomie durch Direktnaht erfolgt und gleichzeitig die Begleitverletzungen therapiert werden.

\section{Wirbelsäulenverletzungen}

Hier ist insbesondere die Relevanz einer situationsgerechten initialen, neurologischen Untersuchung hervorzuheben, um neurologische Defizite zu erkennen. Wird das Kind intubiert und beatmet eingeliefert, muss auf Informationen des Notarztes zurückgegriffen werden.

\section{HWS-Verletzungen}

in einer 2. Operation die endgültige Versorgung der verletzten Lebergefäße und Gallengänge durchgeführt.

Hohlorganverletzungen sind schwierig zu erkennen, daher müssen bei nicht ausschließbarem Verdacht engmaschige klinische Untersuchungen und ggf. auch eine wiederholte CT-Untersuchung mit parenteraler und enteraler Kontrastmittelgabe erfolgen. Werden Hohlorganverletzungen primär erkannt, sind diese meist durch Primärnaht oder Segmentresektion gut zu therapieren. In einem aktuellen Review mit Metaanalyse zu abdominellen Verletzungen konnte gezeigt werden, dass Kinder, die erst im Verlauf mit Anzeichen einer Peritonitis auffielen und sekundär einer Operation zugeführt wurden, ähnlich gute Ergebnisse hatten. Auch wurden diese Kinder meist innerhalb der ersten 24 Stunden symptomatisch. Entscheidend ist in diesem Zusammenhang die engmaschige intensivmedizinische Überwachung mit regelmäßiger klinischer Reevaluation.

Liegt eine stärkere abdominale Kontamination oder eine Rektumverletzung (z.B. durch eine Pfählungsverletzung) vor, ist ein zweizeitiges Vorgehen mit passagerer Anlage eines Anus praeter indiziert.

Bei Beckenverletzungen muss immer an eine Blasenruptur gedacht werden, die eine Indikation zur Frühoperation darstellt.

Pankreasverletzungen sind beim Kind selten und fallen meist erst sekundär bei entsprechendem klinischem und laborchemischem Verdacht auf. Bei ausgedehnten Läsionen sind frühe partielle Resektionen indiziert, die laut
Insbesondere der kraniale Abschnitt der HWS (CO-C2) ist bei Kindern unter 12 Jahren aufgrund des Missverhältnisses zwischen großem Kopf und schwacher Hals-/ Nackenmuskulatur stark verletzungsgefährdet.

Atlantookzipitale Dislokationen gehen meist mit einem schweren Schädel-Hirn-Trauma und einer sehr schlechten Prognose einher. Die Kinder sind oft bereits am Unfallort reanimationspflichtig bzw. versterben am Unfallort. Hier ist das Erreichen einer Stabilität mit Anlage eines Stiff Neck schon am Unfallort entscheidend. Erreichen die Kinder die Klinik, so versterben sie in 50\% der Fälle innerhalb der ersten Stunden.

Bei Kindern unter 12 Jahren können darüber hinaus atlantoaxiale Dislokationen beobachtet werden. Hierbei muss zwischen translatorischen und rotatorischen Instabilitäten des Atlas gegen den Axis unterschieden werden.

C2-/Densfrakturen sind selten und entsprechen üblicherweise Salter-Harris-I-Frakturen. Sie gehen selten mit neurologischen Defiziten einher. Dislozierte Densfrakturen lassen sich in milder Extension verbunden mit einer leichten Translation nach dorsal reponieren.

Therapeutisch ist das konservative Vorgehen mit geschlossener Reposition und anschließender Ruhigstellung im Halo-Fixateur die Methode der Wahl. Operativ kommt eine dorsale Fusion von Okziput und C1/C2 infrage.

Bei Kindern betreffen traumatische ligamentäre Instabilitäten im HWS-Bereich vorwiegend das Segment C2/C3. 


\section{Pädiatrische Orthopädie und Unfallchirurgie}

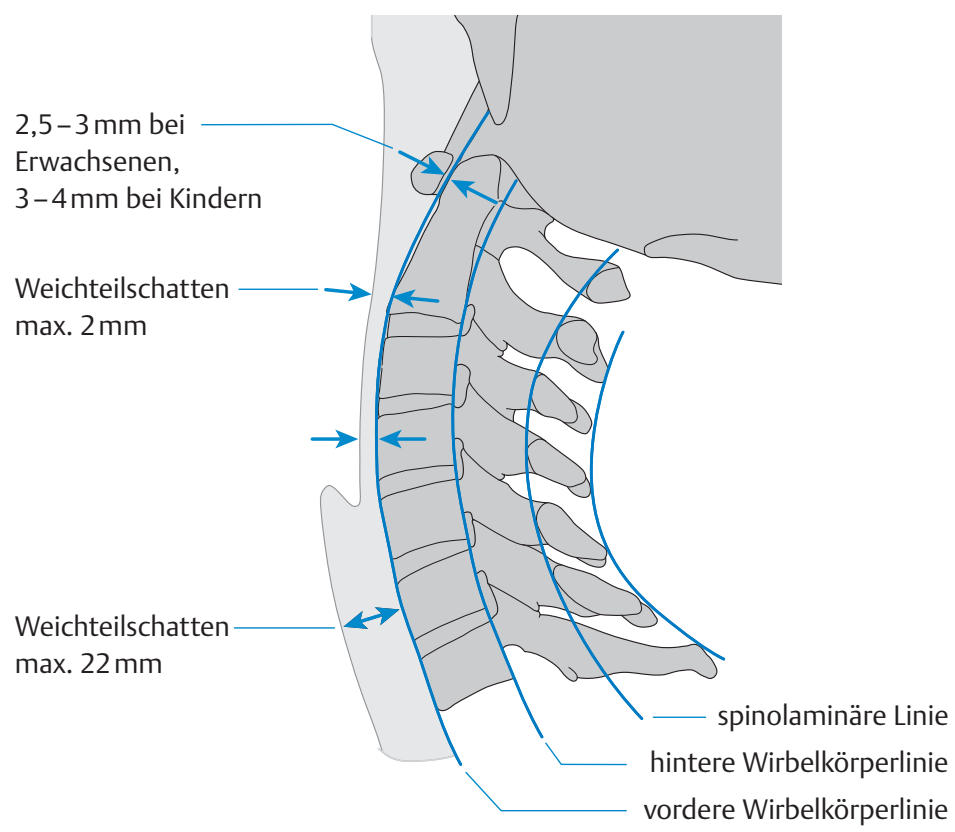

Abb. 6 - Schematische Darstellung einer HWS mit gedachten Hilfslinien zur Beurteilung des Wirbelsäulenalignments. Der Abstand zwischen Dens und Atlasring kann beim Kind bis zu $4 \mathrm{~mm}$ betragen [1].

Methode der Wahl ist auch hier die konservative Therapie mit geschlossener Reposition und Ruhigstellung im Halo-Fixateur für ca. 8 Wochen. Indikation zum operativen Vorgehen besteht bei persistierender C2/C3-Subluxation oder Pseudarthrose. Es erfolgt dann eine dorsale Fusion C1-C3.

Bei der Beurteilung des Wirbelsäulenalignments ist zu beachten, dass der Abstand zwischen Dens und Atlasring beim Kind bis zu $4 \mathrm{~mm}$ betragen kann (Abb. 6).

Physiologische Subluxation bei Kleinkindern bis 6 Jahre!

Verletzungen im Bereich von C3 - C7 kommen selten bei Kindern unter 10 Jahren vor, das Durchschnittsalter beträgt 13 Jahre. Es handelt sich häufig um Kompressionsfrakturen des Wirbelkörpers, Frakturen im Bereich der Facettengelenke und Dislokationen, die durch eine Hyperextension verursacht wurden. Kompressionsfrakturen heilen meist konservativ unter Ruhigstellung im Philadelphia-Kragen aus. Die Spontankorrekturpotenz ist im Vergleich zur BWS/LWS eingeschränkt. Es besteht die Gefahr von Schwanenhalsdeformitäten und kyphotischen Fehlstellungen. Bei persistierender Instabilität, neurologischen Ausfällen und zunehmender Kyphose besteht bei jüngeren Kindern die Indikation zur dorsalen Drahtfusion der Dornfortsätze, da eine ventrale Fusion wegen der Endplattenzerstörung kontraindiziert ist. Bei Jugend- lichen sind ventrale überbrückende Spondylodesen angezeigt.

- Cave: Ventrale Fusionen bei Kleinkindern sind wegen der Endplattenzerstörung zu vermeiden.

\section{BWS- und LWS-Verletzungen}

In 90\% der Fälle werden reine Kompressionsverletzungen beobachtet. Diese werden in erster Linie konservativ therapiert. Eine bleibende kyphotische Fehlstellung kommt bei Kindern unter 12 Jahren ohne neurologische Störungen sehr selten vor, da die Wachstumszone unversehrt ist und die Deformität ausgeglichen werden kann. Bei jüngeren Kindern treten daher kaum langfristige Beschwerden auf.

\section{- Konservative Behandlung von Frakturen der BWS und LWS}

Indikation. Therapie der Wahl bei Typ-A-Frakturen ohne Neurologie.

\section{Therapie}

- kurzzeitige Bettruhe

- Orthese nur in Ausnahmefällen

- schmerzabhängige Mobilisation nach Bettruhe

- Physiotherapie

- Keilwirbel und ossäre Chance-Frakturen sind in speziellen Korsetts behandelbar.

\section{- Operative Behandlung von Frakturen der BWS und LWS}

\section{Indikationen}

- Kompression Wirbelkörper zentral $>50 \%$, lateral $>15^{\circ}$

- Berstungsfrakturen bei Kindern über 12 Jahren, da ansonsten progrediente Kyphosierung droht

- neurologische Defizite

- Typ-B-Verletzungen mit vorwiegend ligamentärer Komponente

- Rotationsverletzungen

\section{Therapie}

- Distraktion und kurzstreckige dorsale Spondylodese durch Fixateur interne oder kleine winkelstabile Implantate

- Dekompression/Laminektomie bei neurologischem Defizit

Die Rolle der ventralen Dekompression und Instrumentation wird derzeit noch kontrovers beurteilt. 
Je älter die Kinder sind und je ausgereifter das Skelett ist, desto eher sollten die für Erwachsene gültigen Versorgungsrichtlinien eingehalten werden.

Ziel der Operation. Ziel einer operativen Therapie ist eine stabile Versorgung der Wirbelsäule, um eine frühe Mobilisation zu ermöglichen und bei kindlichen Polytraumata Lagerungsfähigkeit herzustellen und lagerungsbedingte Sekundärschäden zu vermeiden.

Typ-B- und -C-Verletzungen der Wirbelsäule sind bei Kindern unter 12 Jahren selten und gehen meist aufgrund der Schwere des zugrunde liegenden Unfallmechanismus mit schweren Thorax- und Abdominaltraumata einher. Hier ist eine operative Therapie anzustreben, wobei primär die intrathorakalen oder intraabdominellen Verletzungen im Vordergrund stehen und Versorgungspriorität haben.

Bei dorsalen Fusionen ist eine Metallentfernung notwendig!

\section{Beckenverletzungen}

Die Beckenfraktur im Kindesalter entsteht durch eine erhebliche Gewalteinwirkung, sodass immer mit Begleitverletzungen gerechnet werden muss. Ein Großteil der Patienten ist polytraumatisiert. Ein begleitendes SchädelHirn-Trauma liegt in über 60\% der Fälle vor.

Somit haben die Sicherung der Vitalfunktionen und die Suche nach Organ- und Weichteilverletzungen erste Priorität.

Perianale Hämatombildungen sind pathognomonisch für Rektumläsionen.

Übersicht

\section{Klinische Zeichen einer Beckenfraktur}

- große oberflächliche Hämatombildung inguinal und im Skrotum (Destot-Zeichen)

- Verringerung des Abstands von Trochanter major und Schambeinhöcker im Vergleich zur Gegenseite bei lateralen Kompressionsfrakturen (Roux-Zeichen)

- tastbare Frakturenden oder Hämatombildung bei der rektalen Untersuchung (Earle-Zeichen)

\section{- Konservative Therapie}

Beckenrandfrakturen werden im Regelfall konservativ behandelt. Nach schmerzadaptierter Ruhigstellung erfolgt eine frühfunktionelle Behandlung mit Entlastung an Unterarmgehstützen für 2-4 Wochen. Eine operative Versorgung durch Schraubenosteosynthese ist nur bei grob dislozierten Frakturen indiziert.

Bei vorderen, geschlossenen Beckenringfrakturen mit einer Dislokation von $<5 \mathrm{~mm}$ sowie Symphysensprengungen mit einer Dislokation von $<1 \mathrm{~cm}$ steht die konservative Therapie im Vordergrund. Es erfolgt eine frühfunktionelle Teilbelastung nach 3-4 Wochen, Vollbelastung erst nach 6-8 Wochen.

\section{- Operative Versorgung}

Eine Operationsindikation besteht bei $>1 \mathrm{~cm}$ dislozierten Frakturen des vorderen Beckenrings sowie $>2 \mathrm{~cm}$ dislozierten Symphysenfrakturen. Organverletzungen oder offene Frakturen werden grundsätzlich operativ behandelt. Im Rahmen der Organversorgung erfolgt dann die Reposition, ggf. eine Periostnaht oder im Symphysenbereich darüber hinaus eine Zuggurtung oder Plattenosteosynthese.

Bei der Beurteilung der Symphysenregion ist die altersabhängig unterschiedliche Symphysenweite zu berücksichtigen (Symphysenweite in den ersten 3 Lebensjahren 10-12 mm, beim Erwachsenen 2-4 mm).

Bei Frakturen vom Typ B und C steht die operative Therapie im Vordergrund. Hierbei muss in jedem Fall auf abdominale oder retroperitoneale Begleitverletzungen geachtet werden, die bei der Therapieentscheidung mitberücksichtigt werden müssen.

An Versorgungsoptionen stehen beim Kind oder Jugendlichen sowohl der Fixateur externe, der beim Kind auch als definitives Behandlungskonzept anzusehen ist, und Plattenosteosynthesen zur Verfügung. Die Implantatgröße muss hierbei natürlich an die Größe des kindlichen Beckens angepasst werden. Für die notfallmäßige Anlage einer Beckenzwinge besteht beim Kind keine Indikation; hier sind konventionelle Maßnahmen wie Wickeln der Beine in Innenrotation ausreichend.

- Cave: Jüngere Untersuchungen haben gezeigt, dass das spontane Korrekturpotenzial der Beckenfrakturen in der Vergangenheit überschätzt wurde und das Risiko signifikanter Langzeitfolgen bei verbleibender Fehlstellung besteht. 


\section{OP-Schritte und Tricks}

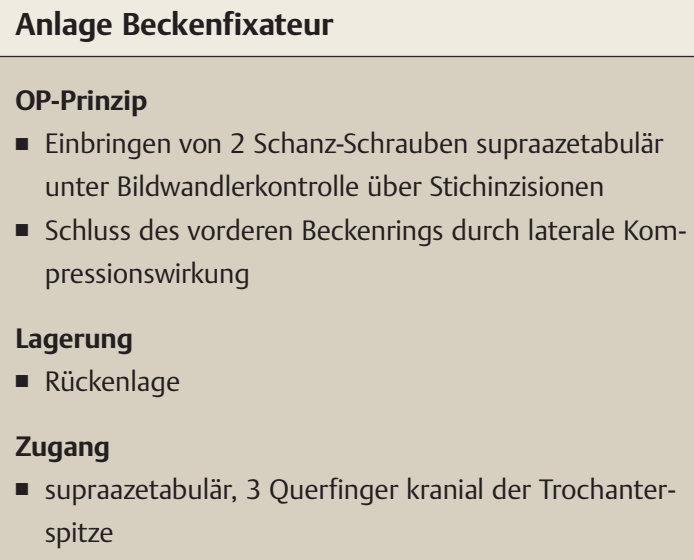

- Einbringen von 2 Schanz-Schrauben supraazetabulär unter Bildwandlerkontrolle über Stichinzisionen

- Schluss des vorderen Beckenrings durch laterale Kompressionswirkung

Lagerung

- Rückenlage

Zugang

- supraazetabulär, 3 Querfinger kranial der Trochanterspitze

Azetabulumfrakturen sind beim Kind sehr selten, daher existieren keine spezifischen Behandlungsalgorithmen. Da bei Fugenverletzungen Wachstumsstörungen resultieren können, ist eine suffiziente Diagnostik erforderlich. Die Therapie muss in Abhängigkeit von der Verletzungsform individuell festgelegt werden. Zur Einschätzung der Dislokation und der Beurteilung der Art der Wachstumsfugenverletzungen sind im Einzelfall MRT-Untersuchungen sinnvoll. Um ein gutes Langzeitergebnis zu erreichen, ist eine anatomische Reposition erforderlich, Methode der Wahl ist die Schraubenosteosynthese. Um das Risiko von Wachstumsstörungen zu vermindern, sollte eine frühzeitige Implantatentfernung erfolgen.

Avulsionsverletzungen sind selten und treten vorwiegend beim jugendlichen Sportler im Alter von 12-16 Jahren auf. Die Therapie ist primär konservativ mit frühfunktioneller Mobilisation (Entlastung an Unterarmgehstützen für 2-4 Wochen) unter adäquater Schmerztherapie.

\section{Extremitätenverletzungen}

Beim polytraumatisierten Kind wird die klassische phasengerechte Versorgung der Frakturen wie beim Erwachsenen nur ausnahmsweise angewendet. Die Unterschiede sollen hier dargestellt werden.

\section{- Akutphase (1. Stunde nach Aufnahme)}

Auch beim Kind stehen in der Akutphase (die 1. Stunde nach Aufnahme, sog. golden Hour of Shock) ausschließlich die lebenserhaltenden Soforteingriffe im Vordergrund. Hierzu gehört im Extremitätenbereich die Stillung arterieller Blutungen. Ansonsten erfolgt eine Ruhigstel- lung von Frakturen sowie die Versorgung von Weichteilverletzungen durch sterile Verbände.

\section{- Primärphase (die ersten 24 Stunden nach Aufnahme)}

In der anschließenden Primärphase sollte die Versorgung dringlicher Extremitätenverletzungen erfolgen. Hierzu zählen

- offene Verletzungen,

- Amputationsverletzungen,

- Kompartmentsyndrome und

- Verletzungen mit Gefäßbeteiligung.

Bei Frakturen mit Kompartmentsyndrom steht die Dekompression der betroffenen Kompartimente im Vordergrund. Eine Weichteildeckung des Implantatmaterials ist grundsätzlich anzustreben. Zur primären Weichteildeckung bei nicht möglichem Wundverschluss bietet sich eine Vakuumversiegelung an. Ein radikales Wunddébridement oder gar die Amputation von Gliedmaßen bei großem Weichteilschaden sollte beim Kind besonders gut überdacht werden.

Erlaubt der Zustand des polytraumatisierten Kindes es, sollten jedoch auch geschlossene Frakturen in der Primärphase bzw. innerhalb der ersten 24-48 posttraumatischen Stunden versorgt werden.

Nach Möglichkeit ist nicht nur eine Stabilisierung, sondern die primäre definitive Versorgung anzustreben, um den Schmerz zu lindern, sekundäre Weichteilschäden zu vermeiden sowie posttraumatischen Stress und Komplikationen zu minimieren.

Für die eher seltenen epi- oder metaphysären Frakturen kommen als Osteosyntheseverfahren die K-Draht-Osteosynthese, ggf. mit Anlage eines gelenküberbrückenden Fixateurs infrage. Die häufiger vorkommenden diaphysären Frakturen können meist durch eine elastisch stabile intramedulläre Nagelung (ESIN o. Ä.; Abb. 7) versorgt werden. Bei größerem Weichteilschaden kommt auch hier der Fixateur externe zur Anwendung. Ab dem Adoleszentenalter werden zunehmend interne schraubenoder plattenosteosynthetische Versorgungstechniken eingesetzt. Die Versorgung orientiert sich dann eher an den für Erwachsene geltenden Prinzipien.

\section{Intermediärphase (2.-5. Tag)}

In der Intermediärphase werden aufgrund der akuten inflammatorischen Reaktionen chirurgische Interventionen soweit möglich vermieden. 

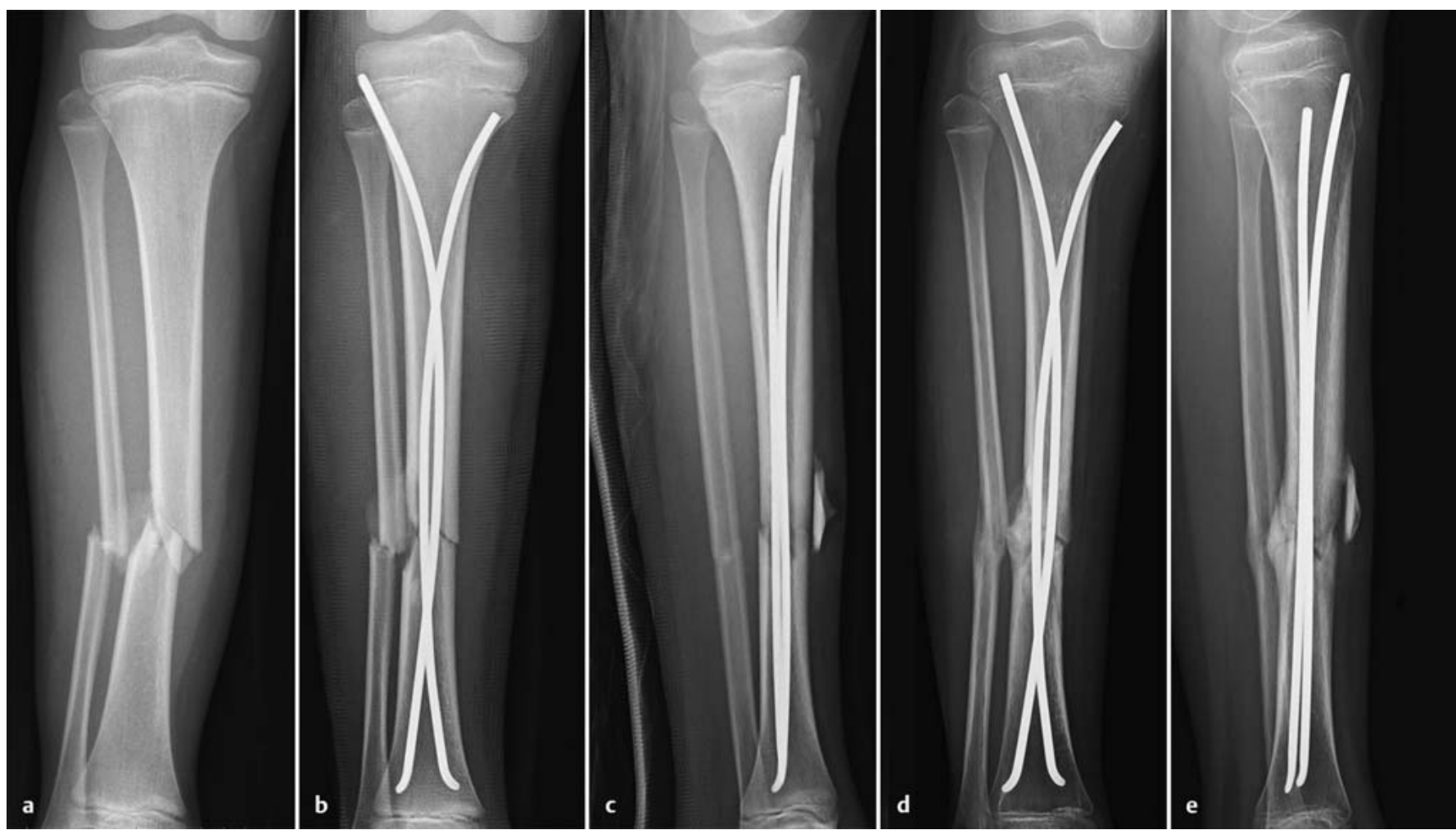

Abb. 7 - 12-jähriger Junge aus Abb. 5, der als Fußgänger angefahren wurde. a Präoperatives Röntgenbild. b Postoperatives Röntgenbild a.-p. nach Versorgung mit ESIN. c Seitliches Röntgenbild postoperativ. d Röntgenbild a.-p. nach Konsolidierung 3 Monate später. e Seitliches Konsolidierungsröntgenbild.

\section{- Sekundärperiode (6. - 14. Tag)}

Die Sekundärperiode dient der Versorgung noch operationsbedürftiger peripherer Frakturen der oberen und unteren Extremität. In dieser Zeit erfolgt die Rekonstruktion komplexer Gelenkverletzungen. Weichteilverletzungen erfordern Revisionseingriffe und rekonstruktive Maßnahmen.

\section{- Tertiärperiode}

Die anschließende Tertiärperiode dient vorwiegend der Mobilisation und Rehabilitation.

\section{Komplikationen}

\section{Schädel-Hirn-Trauma}

Frühkomplikationen beim polytraumatisierten Kind sind in erster Linie durch die Schwere des Schädel-Hirn-Traumas bedingt. Kinder mit einem schweren SHT und einer initialen GCS < 8 entwickeln doppelt so häufig eine diffuses Hirnödem wie Erwachsene. Eine sekundäre neurologische Verschlechterung ist somit häufig nicht durch eine intrakranielle Blutung, sondern die Entstehung eines diffusen Hirnödems bedingt. Schädel-Hirn-traumatisierte Patienten neigen darüber hinaus zur Entwicklung von Thrombosen, sodass auch beim Kind eine adäquate Thromboseprophylaxe erfolgen sollte.

$30 \%$ aller Kinder mit einer initialen GCS $<8$ versterben, $16 \%$ entwickeln ein apallisches Syndrom oder zeigen schwere Beeinträchtigungen. Insgesamt 52\% der Kinder zeigen eine gute Erholungstendenz.

\section{Thoraxtrauma}

Infolge von Lungenkontusionen können auch bei Kindern frühzeitig Komplikationen wie ARDS oder Pneumonien auftreten, sie sind jedoch deutlich seltener als bei Erwachsenen.

Da 20-30\% der Kinder mit schweren Lungenkontusionen im Verlauf eine Pneumonie entwickeln, wird eine frühzeitige antibiotische Therapie empfohlen. 


\section{Abdominaltrauma}

\section{[ Frühkomplikationen}

Die frühen Komplikationen des Abdominaltraumas sind in erster Linie durch den infolge des massiven Blutverlusts ausgelösten hämorrhagischen Schockzustand bedingt. Der Blutverlust führt zu einer Vasokonstriktion mit entsprechender Organminderperfusion. Bei nicht ausreichender Substitutionstherapie resultiert dann eine Organschädigung mit nachfolgendem Organversagen.

Bei Darmperforationen oder protrahierten Darmwandnekrosen entstehen Peritonitiden, die gerade bei sedierten Patienten möglicherweise erst verzögert diagnostiziert werden. Hinzu kommt, dass gerade bei Kindern auch bei höhergradigen Verletzungen von Milz und Leber ein konservatives Prozedere angestrebt wird, sodass Darmverletzungen übersehen werden können.

Zudem können konservativ therapierte Kinder, die zunächst kreislaufstabil waren, sehr rasch bei Zunahme des Blutverlusts kreislaufinsuffizient werden, wodurch ein unverzügliches operatives Eingreifen erforderlich wird. In diesem Zusammenhang sind auch zweizeitige Organrupturen zu erwähnen, sodass bereits bei geringeren intraabdominell freien Flüssigkeitsmengen eine konsequente Kreislaufüberwachung notwendig ist.

$\mathrm{Zu}$ den möglichen postoperativen Komplikationen gehören wie beim Erwachsenen darüber hinaus Nachblutungen, Infekte, Abszesse in parenchymatösen Organen, Nahtinsuffizienzen, Adhäsionen mit Ileuszuständen, Bauchdeckeninfekte und spätere Narbenhernien.

\section{- Spätkomplikationen}

Spätkomplikationen sind zumeist durch übersehene Verletzungen nach stumpfen Bauchtraumata bedingt. Peritonitiden nach übersehenen Darmläsionen machen häufig eine - wenn auch passagere - Anus-praeter-Anlage erforderlich. Zudem erhöht sich das Risiko der Entwicklung einer Sepsis mit resultierendem Multiorganversagen.

\section{Wirbelsäulentrauma}

Wirbelsäulenverletzungen bei Kindern haben insgesamt eine gute Prognose, da posttraumatische Keilwirbelbildungen ausgeglichen werden können. Bei Verletzungen der Wachstumsfugen oder posttraumatischen Lähmungen sind allerdings Wachstumsstörungen und Deformitäten möglich. Verletzungen der Endplatten und Band- scheiben führen meist zur Spontanfusion des Segments. Eine persistierende Instabilität kann allerdings insbesondere bei begleitender neurologischer Störung zur progressiven Deformität führen.

Instabile Verletzungen führen darüber hinaus in einem hohen Prozentsatz zu neurologischen Störungen, wobei es sich in 19-25\% der Fälle um komplette Querschnittsyndrome handelt. Schwere Verletzungen der HWS weisen mit 22-30\% neurologischer Komplikationen das höchste Risiko auf.

Die Prognose der Wirbelsäulenverletzungen korreliert mit der primären Schwere der neurologischen Ausfälle. Etwa 90\% der Kinder mit inkompletter Neurologie verbessern sich. Thorakale Rückenmarkschäden (nach Adamkiewicz [11]) sind in ihrer Prognose am schlechtesten.

\section{Beckentrauma}

\section{- Frühkomplikationen}

Frühkomplikationen bei komplexen Beckenverletzungen sind in erster Linie durch den Blutverlust infolge der Begleitverletzungen bedingt, wobei eine rasche Abgrenzung zwischen intraabdomineller und retroperitonealer Blutung wesentlich ist. Die Behandlung intraabdomineller Verletzungen erfolgt nach den bekannten Richtlinien der Abdominalchirurgie.

Problematischer ist die Therapie retroperitonealer Blutungen. Massive retroperitoneale Blutungen aus den frakturierten spongiösen Knochen oder dem präsakralen Venenplexus sind wesentlich schwieriger zu stillen und stellen infolge des massiven hämorrhagischen Schocks mit den nachfolgenden Organkomplikationen eine mögliche frühzeitige Todesursache dar. Zur aktiven Kontrolle einer retroperitonealen Blutung bei instabilen Beckenringverletzungen wird daher, wie beim Erwachsenen, eine Frühstabilisierung des Beckens durch einen Fixateur externe empfohlen. Hierdurch kann eine Minderung von Blutungen aus dem Knochen und dem venösen Plexus erreicht werden. Ist nach Notfallstabilisierung des Beckens keine Kreislaufstabilisierung zu erreichen, so wird auch im Kindesalter eine Angiografie mit Möglichkeit der Embolisation empfohlen.

Insbesondere bei Beckenfrakturen mit Zerreißung des Beckenbodens sowie Läsion von Anus und Rektum besteht das Risiko der Entwicklung einer Durchwanderungsperitonitis mit septischem Krankheitsbild. Derartige Verletzungen erfordern eine frühzeitige 
Rekonstruktion der Weichteile mit Anlage eines protektiven Anus praeter sowie die Entfernung von Kot und Blutresten aus dem Rektum.

\section{- Spätkomplikationen}

Trotz frühzeitiger Reposition und Stabilisierung können bei Beckenringfrakturen Typ B und C gravierende Wachstumsstörungen als Folge von Wachstumsfugenverletzungen durch Fusion der Symphyse oder Iliosakralfugen auftreten.

Auch Azetabulumfrakturen können insbesondere bei Stauchung der Cartilago triradiata (Salter-Harris V) zu schwerwiegenden Wachstumsstörungen bis zur Aplasie einer Beckenhälfte oder Dysplasie des Azetabulums mit konsekutiver Hüftluxation führen.

\section{Extremitätentrauma}

\section{- Frühkomplikationen}

Bei Kindern kann sich - wie bei Erwachsenen - nach einem Weichteiltrauma oder einer Fraktur ein Kompartmentsyndrom entwickeln, das frühzeitig erkannt und operativ therapiert werden muss.

Plastische Deckungen nach Weichteilverletzungen sind selten notwendig, da eine hohe Regenerationsfähigkeit des Gewebes besteht und lokale Plastiken häufig erfolgreich sind. Auch bei Kindern haben sich temporäre Weichteildeckungen mit VAC-Systemen bewährt.

\section{- Spätkomplikationen}

Gerade beim Adoleszenten können im Bereich der langen Röhrenknochen Pseudarthrosen auftreten, die radiologisch erkannt und entsprechend therapiert werden müssen.

Die Beteiligung der Wachstumsfugen kann beim Kind zu einem partiellen oder generalisierten frühzeitigen Fugenschluss und hierdurch letztendlich zu einem Fehlwachstum der betroffenen Extremität führen. Beinlängendifferenzen müssen ebenfalls im Wachstumsverlauf erkannt und mit entsprechenden konservativen oder operativen Maßnahmen ausgeglichen werden. Die Spontankorrekturfähigkeit von Achsabweichungen und Rotationsfehlern ist altersabhängig. Die Kinder müssen dementsprechend regelmäßig nachuntersucht und klinisch relevante Abweichungen zu einem günstigen Zeitpunkt korrigiert werden.

\section{Nachbehandlung}

\section{Schädel-Hirn-Trauma}

War infolge eines Schädel-Hirn-Traumas eine temporale Kraniektomie erforderlich, wird der Kalottendeckel konserviert und üblicherweise nach Abschwellen der Hirnmasse nach 3-6 Monaten replantiert. Darüber hinaus sollten Kinder mit einem relevanten Schädel-Hirn-Trauma möglichst frühzeitig neurologischen Rehamaßnahmen zugeführt werden.

\section{Thoraxtrauma}

Thoraxtraumata werden in erster Linie konservativ unter entsprechender physiotherapeutischer Betreuung nachbehandelt.

\section{Abdominaltrauma}

Nach Abdominaltraumata sind auch im Kindesalter intraabdominelle Adhäsionen nicht auszuschließen, sodass im Verlauf auf weiche Stuhlgänge und regelmäßige Darmentleerungen zu achten ist. War die Anlage eines doppelläufigen protektiven oder rückverlagerungsfähigen endständigen Anus praeter erforderlich, kann dieser üblicherweise 3 Monate nach dem Trauma zurückverlagert werden.

\section{Wirbelsäulentrauma}

Bei Wirbelsäulenverletzungen ist ebenfalls möglichst frühzeitig nach operativer Stabilisierung eine neurologische Rehabilitation indiziert.

Die Entfernung eines Halo-Fixateurs erfolgt 8-12 Wochen nach Anlage. Interne Osteosynthesen im Wirbelsäulenbereich werden altersabhängig nach etwa 1 Jahr entfernt.

Radiologische Kontrollen im Wirbelsäulenbereich sind regelmäßig erforderlich: postoperativ nach 6 , nach 12 Wochen und nach 1 Jahr. Gerade im oberen HWSBereich sollte zur besseren Beurteilung der Durchbauung eine CT-Kontrolle erfolgen. Bei klinisch relevanten Skoliosen, insbesondere bei begleitender neurologischer Störung, sind regelmäßige Kontrollen im gesamten Wachstumsverlauf erforderlich, um progressive Deformitäten mit entsprechender Beschwerdesymptomatik zu erkennen. 


\section{Beckentrauma}

Der Fixateur externe bei Beckenverletzungen ist je nach Verletzungstyp beim Kind als endgültiges osteosynthetisches Verfahren anzusehen, sodass hier nicht zwingend Folgeoperationen erforderlich sind. Eine Fixateurentfernung wird nach ca. 3-6 Wochen empfohlen, solange ist keine Vollbelastung möglich. Implantate, die die Iliosakralfugen kreuzen oder die Symphyse überbrücken, sollten nach 3-6 Monaten entfernt werden.

- Essenziell in der Nachbehandlung von Kindern ist ebenso wie beim Erwachsenen eine suffiziente Schmerztherapie.

\section{Prognose}

Outcome und Mortalitätsrate werden letztendlich in bis zu 90\% der Fälle durch die Schwere des Schädel-HirnTraumas bestimmt.

- Ein signifikant erhöhtes Letalitätsrisiko besteht für polytraumatisierte Kinder mit einem ISS $\geq 25$, einem GCS-Score $<8$ und einer Notfallbluttransfusion von $\geq 20 \mathrm{ml} / \mathrm{kg}$ Körpergewicht.

Die Letalität ist nicht abhängig von Altersgruppen. Die Hälfte aller Todesfälle ereignet sich innerhalb der ersten 72 Stunden nach Trauma.

Ein weiterer wichtiger prognostischer Parameter ist der intrakranielle Druck. Bei einem ICP zwischen 20 und $40 \mathrm{mmHg}$ beträgt die Letalität $28 \%$, bei einem dauerhaften ICP über $40 \mathrm{mmHg}$ beträgt die Letalität $100 \%$. Ein schweres Schädel-Hirn-Trauma, ein diffuses Hirnödem und ein akutes subdurales Hämatom erhöhen das Risiko von zerebralen Krampfanfällen. Die Anfallshäufigkeit korreliert darüber hinaus mit der initialen GCS und nimmt mit zunehmender GCS ab.

Bei Entlassung aus der stationären Behandlung zeigt ein Großteil der Kinder in Abhängigkeit von der Schwere des Schädel-Hirn-Traumas und der Extremitätenverletzungen kognitive und physische Beeinträchtigungen. Die Hauptursache für Behinderungen in den ersten Wochen nach dem erlittenen Trauma sind neurologische Defizite wie Spastik, Sprach- oder Sehstörungen als Folge des SchädelHirn-Traumas. Myelonschädigungen führen zudem in Abhängigkeit von der Höhe der Schädigung zu entsprechenden sensiblen oder motorischen Störungen bis hin zum irreversiblen Querschnitt.
Der überwiegende Anteil der polytraumatisierten Kinder wird vom Krankenhaus aus direkt in speziell für pädiatrische Patienten ausgestattete Rehaeinrichtungen verlegt. Gerade bei Kindern und Jugendlichen führt eine intensive Rehabilitationstherapie, verbunden mit schulischer oder beruflicher Förderung, zu einer deutlichen Rückbildung der primär vorliegenden zerebralen oder physischen Einschränkungen. Sechs Monate nach dem Trauma zeigt ein Großteil der pädiatrischen Patienten eine deutliche Verbesserung im Outcome.

\section{Begutachtung}

Die Begutachtung kindlicher Körperstammverletzungen erfolgt nach den für Erwachsene gültigen Richtlinien. Sind Wachstumsstörungen zu erwarten, müssen entsprechende Nachbegutachtungen durchgeführt werden. Neurologische Defizite nach Schädel-Hirn-Traumata, Wirbelsäulen-, Becken- oder Extremitätenverletzungen erfordern eine entsprechende Zusatzbegutachtung. Ebenso dürfen gerade bei polytraumatisierten Patienten psychische Begleitreaktionen nicht außer Acht gelassen werden. Auch hier ist ggf. eine Zusatzbegutachtung zu empfehlen.

\section{Perspektiven}

Aufklärung und Prävention, aber auch die Einführung von Helmen für Fahrradfahrer, die Anschnallpflicht, die Verwendung altersgerechter Kindersitze mit entsprechender Anschnallpflicht führten zu einer Verbesserung der Prognose kindlicher Schädel-Hirn- und Körperstammverletzungen.

Kinder sind keine „kleinen Erwachsenen“, sodass eine Versorgung von polytraumatisierten Kindern in Traumazentren mit Erfahrung in Kindertraumatologie erforderlich ist. Eine pädiatrisch orientierte Intensivstation, Z.B. in einem angeschlossenen Zentrum für Kinder- und Jugendmedizin, ist zu fordern, da gerade die Intensivtherapie des Kindes andere Strategien erfordert als die bei erwachsenen Patienten.

Auch in Zukunft müssen Behandlungsstrategien multizentrisch ständig neu evaluiert und verbessert werden, um eine Vereinheitlichung der Maßnahmen zu erreichen.

Ab 2016 ist durch die Sektion Kindertraumatologie der DGU die Erstellung einer S2-Leitlinie für die Versorgung schwer verletzter Kinder vorgesehen, die im Jahr 2017 fertiggestellt werden soll. 
Interessenkonflikt: Die Autoren geben an, dass kein Interessenkonflikt besteht.

\section{Literatur}

1 Magerl F, Aebi M, Gertzbein SD et al. A comprehensive classification of thoracic and lumbar injuries. Eur Spine J 1994; 3: 184-201

2 Blauth M, Tscherne H. Wirbelsäule. Berlin: Springer; 1998

3 Jakob H, Marzi I. Management des schwer verletzten Kindes. Orthop Unfallchir up2date 2007; 2: 79-100

4 Pennal GF, Tile M, Waddell JP et al. Pelvic disruption - assessement and classification. Clin Orthop 1980; 151: 12-21

5 Salter R, Harris W. Injuries involving the epiphyseal plate. J Bone Joint Surg Am 1963; 45: 587-622

6 Salter RB. Injuries of the epiphyseal plate. Instr Course Lect 1992; 41: $351-359$

7 Aitken AP. Fractures of the epiphyses. Clin Orthop Relat Res 1965; 41 : $19-23$

8 Slongo TF, Audigé L, AO Pediatric Classification Group. Fracture and dislocation classification for children: the $\mathrm{AO}$ pediatric comprehensive classification of long bone fractures (PCCF). J Orthop Trauma 2007; 21: 135 - 160

9 Slongo T, Audigé L, Clavert JM et al. The AO comprehensive classification of pediatric longbone fractures: a web-based multicenter agreement study. J Pediatr Orthop 2007; 27: 171 - 180

10 Linhart WE. Li-La Licht und Lachen für kranke Kinder. Effizienz in der Medizin e. V. 8/2009. Im Internet: http://www.li-la.org

11 Adamkiewicz A. Die Blutgefäße des Menschlichen Rückenmarks. Wien: Sitz Ber Acad Wiss, Math Nat KI 1881, 1882; 84: 469 und 85: 101

\section{Zum Weiterlesen und Vertiefen}

Auner B, Marzi I. Pediatric multiple trauma. Chirurg 2014; 85: 451 - 464 Debus F, Lefering R, Frink $M$ et al. Das Polytrauma von Kindern und Jugendlichen. Orthop Unfallchir Praxis 2013; 12: 565- 571

Dietz H-G, Illing P, Schmittenbecher P et al. Praxis der Kinder- und Jugendtraumatologie. Berlin: Springer; 2011

Jakob H, Brand J, Marzi I. Das Polytrauma im Kindesalter. Unfallchirurg 2009; 112: 951 - 958

Jakob H, Wyen H, Marzi I. Polytrauma im Kindesalter. Trauma Berufskrankh 2013; 15: 67 - 74
Von Laer L, Kraus R, Linhart W. Frakturen und Luxationen im Wachstumsalter. 6. Aufl. Stuttgart: Thieme; 2012

Landau A, van As AB, Numanoglu A et al. Liver injuries in children: the role of selective non-operative management. Injury 2006; 37: 66-71

Laurer HL, Wutzler S, Wyen H et al. Quality of prehospital and early clinical care of pediatric trauma patients of school age compared to an adult cohort. A matchedpair analysis of 624 patients from the DGU trauma registry. Unfallchirurg 2009; 112: 771 - 777

Löllgen R, Szabo L. Schock im Kindesalter. Med Klin Intensivmed Notfmed 2015; 110: 338

Marzi I. Kindertraumatologie. 3. Aufl. Berlin: Springer; 2016

Marzi I, Rose S. Praxisbuch Polytrauma. 1. Aufl. Köln: Deutscher ÄrzteVerlag; 2012: 1-397

Nau C, Rose S, Laurer HL et al. Wirbelsäulenverletzungen im Kindesalter. Orthopäd Unfallchir up2date 2010; 5: 23-38

Patel N, West M, Wurster ] et al. Pediatric traumatic brain injuries treated with decompressive craniectomy. Surg Neurol Int 2013; 4: 128

Schmittenbecher PP. Stumpfes Bauchtrauma. Monatsschr Kinderheilkd 2013; 161: 122 - 130

Schneidmüller D, Wutzler S, Kelm A et al. Beckenverletzungen im Kindesund Jugendalter: Eine retrospektive Analyse eines überregionalen Traumazentrums über 5 Jahre. Unfallchirurg 2011; 114: 510 - 516

Schöneberg C, Schweiger B, Metzelder M et al. Das verletzte Kind diagnostisches Vorgehen im Schockraum. Unfallchirurg 2014; 117 $829-841$

Schöneberg C, Tampier S, Hussmann B et al. Diagnostik des stumpfen Abdominaltraumas des Kindes - ein systematisches Review mit Metaanalyse. Zentralbl Chir 2014; 139: 584-591

Slongo TF. Acetabulum injuries in infancy and childhood. Unfallchirurg 2013; 116: 1076 - 1084

Strohm PC, Schmittenbecher PP. Fracture stabilization in polytraumatized children. Unfallchirurg 2011; 114: 323-332

Voth M, Nau C, Marzi I. Thoracic and lumbar spinal injuries in children and adolescents. Unfallchirurg 2013; 116: 1062 - 1068

Wyen $\mathrm{H}$, Jakob $\mathrm{H}$, Wutzler $\mathrm{S}$ et al. Prehospital and early clinical care of infants, children, and teenagers compared to an adult cohort. Eur ] Trauma Emerg Surg 2010; 36: 300 - 307

\section{Korrespondenzadresse}

Dr. B. Auner

Klinik für Unfall-, Hand- und Wiederherstellungschirurgie Universitätsklinikum Frankfurt

Goethe-Universität

Theodor-Stern-Kai 7

60590 Frankfurt am Main

E-Mail: birgit.auner@kgu.de 


\section{Pädiatrische Orthopädie und Unfallchirurgie}

\section{CME-Fragen}

\author{
Wodurch ist das Thoraxtrauma \\ beim polytraumatisierten \\ Kind gekennzeichnet?
}

\section{CME•thieme.de}

\section{CME-Teilnahme}

- Viel Erfolg bei Ihrer CME-Teilnahme unter http://cme.thieme.de

- Bitte informieren Sie sich vorab online über die Gültigkeitsdauer.

- Sollten Sie Fragen zur Online-Teilnahme haben, unter http://cme.thieme.de/hilfe finden Sie eine ausführliche Anleitung.
Eine der folgenden Aussagen

zu Extremitätenfrakturen

beim polytraumatisierten Kind ist unzutreffend. Welche?
A Ein unauffälliges Röntgenbild schließt ein Thoraxtrauma aus.

B Eine Lungenkontusion kommt bei Kindern selten vor.

C Eine Rippenserienfraktur kommt bei Kindern selten vor.

D Wenn äußerlich keine Verletzungszeichen zu sehen sind, ist eine Verletzung innerer Strukturen sehr unwahrscheinlich.

E Ein Pneumothorax muss im Gegensatz zu Erwachsenen bei Kindern nur selten entlastet werden.
Welche Aussage zum

Abdominaltrauma beim polytraumatisierten Kind ist korrekt?
A Mehrfragmentäre Extremitätenfrakturen beim polytraumatisierten Kind sind selten.

B Die beim erwachsenen Patienten übliche phasengerechte Versorgung der Extremitätenfraktur kommt beim pädiatrischen Patienten nur ausnahmsweise zur Anwendung.

c Extremitätenfrakturen beim polytraumatisierten Kind finden sich am häufigsten epi-/metaphysär.

D Bei V.a. Extremitätenfraktur erfolgt die Röntgenuntersuchung inkl. des angrenzenden Gelenks bzw. der angrenzenden Gelenke.

E Extremitätenfrakturen beim polytraumatisierten Kind treten häufig als Begleitverletzung eines schweren Schädel-Hirn-Traumas auf. 
Wodurch ist das Schockraummanagement bei Kindern gekennzeichnet?
A Eine standardisierte, algorithmenbasierte Schockraumversorgung ist bei Kindern nicht sinnvoll.

B Um unnötige Manipulationen zu vermeiden, kann auf die Beurteilung der peripheren Durchblutung, Motorik und Sensibilität beim Kind verzichtet werden.

C Da die Anamnese bei Kindern häufig ungenau ist, kann auf diese auch zur Beurteilung der Vigilanz verzichtet werden.

D Das Schockraumteam sollte durch einen Pädiater oder pädiatrischen Intensivmediziner erweitert werden.

E Für die Verlegung in ein geeignetes Traumazentrum existieren in Deutschland keine Verlegungskriterien.
Über den Schockraum Ihrer Klinik wird ein 8-jähriger Junge eingeliefert, der als Rollerfahrer beim Überqueren der Straße von einem Pkw angefahren wurde und etwa 5-6 Meter durch die Luft geflogen ist. Es besteht der Verdacht auf ein Becken- und Thoraxtrauma. Laut Übergabe des Primärnotarztes habe am Unfallort ein GCS von 15 Punkten bestanden. Es erfolgte die Intubation bei V.a. schwere Mehrfachverletzung für den Transport per RTH. Sie überlegen nun, ob eine CT-Untersuchung des Schädels im Rahmen der Diagnostik indiziert ist. Welche Aussage trifft in diesem Zusammenhang zu?
A Bei einem initialen GCS von 15 Punkten laut Übergabe des Primärnotarztes kann auf eine CCT verzichtet werden.

B Eine CCT sollte erfolgen, da der Primärnotarzt nicht vor Ort ist und mit einem Informationsverlust gerechnet werden muss.

C Der Unfallmechanismus schließt eine Kopfverletzung aus.

D Eine CCT-Untersuchung sollte beim intubierten Kind und entsprechenden Unfallmechanismus obligat erfolgen.

E Eine CCT-Untersuchung kann sekundär erfolgen, wenn das Kind post extubationem neurologisch auffällig erscheint.
Welche Aussage zu schweren Verletzungen im Säuglingsalter trifft nicht zu?

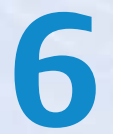

A Ein häufiger Unfallmechanismus ist der Sturz vom Wickeltisch.

B Das Risiko, durch Gewalteinwirkung zu sterben, ist bei Säuglingen im Vergleich zu älteren Kindern eher gering.

C Das Schädel-Hirn-Trauma ist die häufigste Todesursache.

D Durch den Abriss einer Brückenvene kann es zu subduralen Hämatomen kommen.

E Epidurale Hämatome sind im Säuglingsalter eher selten.
Nur eine der folgenden Aussagen zum Polytrauma bei Kindern unter 15 Jahren trifft zu. Welche?

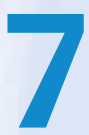

A Das Polytrauma in dieser Altersgruppe ist häufig.

B Die Verletzungsmuster und -ursachen sind nicht vom Alter abhängig.

C Jungen sind häufiger betroffen.

D Ein Suizidversuch ist als Todesursache ausgeschlossen.

E Die physiologischen Besonderheiten beim Kind können in der Notfallsituation vernachlässigt werden. 


\section{Pädiatrische Orthopädie und Unfallchirurgie}

Welche Aussage zu

Beckenfrakturen beim poly-

traumatisierten Kind trifft zu?

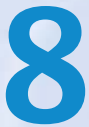

A Je jünger die Kinder sind, desto einfacher lassen sich Beckenfrakturen diagnostizieren.

B Begleitverletzungen des Urogenitaltrakts und des Rektums liegen nur selten vor.

C Bei Verletzungen der Wachstumszonen sind Wachstumsstörungen im weiteren Verlauf nicht zu erwarten.

D Beim Vorliegen einer Beckenfraktur muss von einer besonderen Schwere der Verletzung ausgegangen werden.

E Bei der operativen Versorgung kommt der supraazetabuläre Fixateur externe bei Kleinkindern nur selten zum Einsatz.
Wodurch ist das Wirbelsäulentrauma beim polytraumatisierten Kind gekennzeichnet?
A Da eine MRT-Untersuchung sehr aufwendig ist, kann beim Vorliegen eines neurologischen Defizits und negativer CT-Untersuchung zunächst der Spontanverlauf abgewartet werden.

B Aufgrund der geringen Masse des kindlichen Körpers und der hohen Elastizität der Wirbelsäule treten Wirbelsäulenfrakturen insgesamt häufig auf.

C Da das Rückenmark beim Kind longitudinale Dehnungen gut toleriert, kommen neurologische Defizite äußerst selten vor.

D Bei Kleinkindern ist aufgrund des ungünstigen Kopf-Körper-Verhältnisses und der schwachen Nackenmuskulatur eher mit einer Verletzung der HWS zu rechnen.

E Komplexe Verletzungen im Bereich des Atlas und des Axis haben eine gute Prognose.
Nur eine der folgenden Aussagen über das schwere Schädel-Hirn-Trauma bei Kindern ist korrekt. Welche?
A Das Schädel-Hirn-Trauma ist nicht entscheidend für die Prognose.

B Eine dekompressive Hemikraniektomie bei raumfordernden Blutungen sollte nur als Ultima Ratio erfolgen.

C Bei Kleinkindern und Säuglingen ist die neurologische Beurteilung per GCS unüblich.

D Bei infolge einer Kopfverletzung somnolenten Kindern sollte eine Intubation möglichst spät erfolgen, um die neurologische Beurteilung zu gewährleisten.

E Ein diffuses Hirnödem entwickelt sich beim pädiatrischen Patienten häufiger als beim Erwachsenen. 